\title{
An Adaptive Self-Interference Cancelation/Utilization and ICA-Assisted Semi-Blind Full-Duplex Relay System for LLHR IoT
}

\author{
Hanjun Duan, Student Member, IEEE, Xu Zhu ${ }^{\circledR}$, Senior Member, IEEE, Yufei Jiang ${ }^{\circledR}$, Member, IEEE, \\ Zhongxiang Wei, Member, IEEE, and Sumei Sun ${ }^{(0}$, Fellow, IEEE
}

\begin{abstract}
In this article, we propose a semi-blind full-duplex (FD) amplify-and-forward (AF) relay system with adaptive self-interference (SI) processing assisted by independent component analysis (ICA) for low-latency and high-reliability (LLHR) Internet of Things (IoT). The SI at FD relay is not necessarily canceled as much as possible like the conventional approaches, but is canceled or utilized based on a signal-to-residual-SI ratio (SRSIR) threshold at relay. According to the selected SI processing mode at relay, an ICA-based adaptive semi-blind scheme is proposed for signal separation and detection at destination. The proposed FD relay system not only features reduced signal processing cost of SI cancelation but also achieves a much higher degree of freedom in signal detection. The resulting bit error rate (BER) performance is robust against a wide range of SRSIR, much better than that of conventional FD systems, and close to the ideal case with perfect channel state information (CSI) and perfect SI cancelation. The proposed system also requires negligible spectral overhead as only a nonredundant precoding is needed for ambiguity elimination in ICA. In addition, the proposed system enables full resource utilization with consecutive data transmission at all time and same frequency, leading to much higher throughput and energy efficiency than the time-splitting and power-splitting-based self-energy recycling approaches that utilize only partial resources. Furthermore, an intensive analysis is provided, where the SRSIR thresholds for the adaptive SI
\end{abstract}

This work was supported in part by the National Natural Science Foundation of China under Grant 61901138, in part by the Natural Science Foundation of Guangdong Province under Grant 2018A030313344 and Grant 2018A030313298, and in part by the Guangdong Science and Technology Planning Project 2018B030322004. This article was presented in part at the IEEE Wireless Communications and Networking Conference (WCNC), Marrakesh, Morocco, Apr. 15-19, 2019. (Corresponding author: Yufei Jiang.)

H. Duan and Y. Jiang are with the School of Electronic and Information Engineering, Harbin Institute of Technology, Shenzhen 518055, China (e-mail: duanhanjun@stu.hit.edu.cn; xuzhu@liverpool.ac.uk).

$\mathrm{X}$. Zhu is with the School of Electronic and Information Engineering, Harbin Institute of Technology, Shenzhen 518055, China, and also with the Department of Electrical and Electronics Engineering, University of Liverpool, Liverpool L69 3GJ, U.K. (e-mail: jiangyufei@hit.edu.cn).

Z. Wei is with the Department of Electrical and Electronic Engineering, University College London, London WC1E 6BT, U.K.

S. Sun is with the Department of Communications and Networks, Institute for Infocomm Research, Agency for Science, Technology and Research, Singapore. processing mode selection and the BER expressions with ICA incurred ambiguities are derived.

Index Terms-Full-duplex (FD) relay, independent component analysis (ICA), low-latency and high-reliability (LLHR) Internet of Things (IoT), self-interference (SI).

\section{INTRODUCTION}

$\mathbf{I}$ NTERNET of Things (IoT) is an emerging technology for connecting a massive number of intelligent devices [1][3]. Low-latency and high-reliability (LLHR) IoT applications, such as industrial automation, virtual and augmented reality, and healthcare are life-changing applications [4], [5]. However, they introduce communication challenges concerning reliability, latency, connectivity, and spectral efficiency (SE) [6]. Full-duplex (FD) transmission has emerged as a promising technique to improve SE and reduce transmission delay [7]. Relaying is a fundamental technique that enables enhanced transmission reliability and extended coverage of wireless networks [8]. Therefore, the research on FD relay networks plays an important role in the development of LLHR IoT.

Compared to half-duplex (HD) relay networks [9], where transmission and reception operate at orthogonal time slots or frequencies, FD relay networks allow simultaneous transmission and reception at same frequency and thus can approximately double the SE over HD relay networks. However, FD transmission introduces strong self-interference (SI) from the transmit antennas to the receive antennas at relay. There are mainly three kinds of SI mitigating approaches, namely, passive suppression (PS) [10]-[12], analog cancelation (AC) [10], [11], [13], and digital cancelation (DC) [14], [15]. PS is a technique to physically separate the transmit chain from the receive chain in propagation domain, via antenna isolation [10], [11], directional antenna, antenna polarization, antenna shielding, etc. [12]. AC schemes generally cancel SI in the analog-receive chain or circuitry by subtracting a copy of the predicted SI from the received signal. AC is implemented in radio frequency (RF) domain to prevent the receiver components saturation [10], [11], or at baseband [13]. DC is applied after PS and AC to subtract the 
residual SI in digital domain [15]. It requires accurate estimation of the residual SI, which is normally hard to obtain. It is now feasible to achieve up to $110 \mathrm{~dB}$ of SI cancelation amount [16]. However, active SI cancelation by $\mathrm{AC}$ and DC requires high power consumption, which could even be comparable with that of transmit/receive chains [17].

To alleviate the high-power consumption at the FD relay node, self-energy recycling has been investigated [18]-[24]. It can harvest energy from the SI by wireless information and power transmission (WIPT) techniques [18], [19]. Existing FD aided self-energy recycling relay systems are based on timesplitting (TSP) [18], [20] or power-splitting (PSP) [21], [22] structures. For the TSP-based FD relay systems [23], two time slots are required, where in the first time slot the source sends energy-bearing signal to the relay for energy harvesting $(\mathrm{EH})$ and in the second time slot, the source sends informationbearing signal to the relay and the relay forwards the received signal to the destination simultaneously. As for the PSP-based FD relay systems [24], the relay splits part of the received signal in analog domain for $\mathrm{EH}$, and forwards the rest signal to destination for decoding. However, the aforementioned work should be classified as pseudo-FD realization due to utilize partial resource (time or power) for wireless power transfer. Also, the previous work has only utilized SI as an energy source, but not used it to enhance the degree of freedom in signal detection.

Independent component analysis (ICA) [25]-[27], a higher order statistics (HOS)-based blind source separation approach, is a spectrum-efficient and effective equalization method, as no training data are required to estimate the channel state information (CSI). ICA has been used for multiple-inputmultiple-output (MIMO) and orthogonal frequency-division multiplexing (OFDM) systems [25]-[27]. It can achieve a performance close to the ideal case with perfect CSI, given effective elimination of quadrant and permutation ambiguities via a few pilots [27] or nonredundant precoding [28].

In this article, we propose a novel ICA-assisted semi-blind FD amplify-and-forward (AF) relay system for LLHR IoT, where consecutive FD data transmission is enabled at all time and same frequency, achieving full resource utilization. At FD relay, we do not aim to cancel SI as much as possible and therefore do not require complex DC of SI. Instead, SI is canceled by PS only or PS $+\mathrm{AC}$ modes. The residual SI is transmitted to destination alongside the desired signal, to enhance the degree of freedom in signal detection or $\mathrm{EH}$. At destination, for relatively low signal-to-residual-SI ratio (SRSIR) case, ICA is used to separate the desired signal and the residual SI, and also detect the desired signal. While for high SRSIR case, a space-splitting (SS)-based approach is used to regroup antennas, where part of receive antennas are used for signal detection and the others for EH. Hence, there are four SI processing modes which can be selected adaptively according to the SRSIR. The mode selection-based adaptive scheme is referred to as ICA-SS. This article is different in the following aspects.

1) The proposed adaptive SI processing mode selection scheme introduces a much higher degree of freedom in signal detection and energy recycling than the previous work [1], [14], [16], and enables a bit error rate (BER) performance that is robust against a wide range of SRSIR. It conducts adaptive signal processing at both relay and destination with four choices of modes in total, while only two SI suppression modes at relay were considered in our previous work [1]. Furthermore, the proposed adaptive SI processing scheme leads to significant reduction in energy and signal processing costs over the conventional methods [14], [16], where SI is canceled as much as possible.

2) The proposed ICA-SS-assisted semi-blind FD AF relay system achieves full resource utilization with consecutive FD data transmission at all time and same frequency. While the TSP [18] and PSP [22]-based self-energy recycling $\mathrm{FD}$ systems utilize only partial time slots or signal power, respectively. The proposed FD relay system significantly outperforms the TSP [18] and PSP [22]-based FD systems in terms of throughput, energy efficiency (EE), and BER.

3) An intensive theoretical analysis is provided. Two SRSIR thresholds are derived for adaptive SI processing mode selection at relay. It is proved that the BER performance of the proposed system is robust against SRSIR. The BER expressions with ICA incurred ambiguities are also derived. While in our previous [1], only throughput and EE were analyzed for an FD relay system with two adaptive SI processing modes at relay. Thanks to the utilization of ICA at destination, the proposed system demonstrates a superior BER performance close to the ideal case with perfect CSI and perfect SI cancelation, and also requires negligible spectral overhead as no training data but a spectrum nonredundant precoding is needed for ambiguity elimination in ICA.

The remainder of this article is organized as follows. The system model is described in Section II. The adaptive SI processing mode selection strategy and the algorithms of ICAassisted signal detection and energy recycling are presented in Sections III and IV, respectively. Performance analysis and complexity analysis are provided in Sections V and VI, respectively. The numerical results are provided in Section VII, and conclusion is drawn in Section VIII.

Notations: Throughout this article, we use bold symbols to represent vectors/matrices. Superscripts $*, \mathrm{~T}, \mathrm{H}$, and $\dagger$ denote complex conjugate, transpose, complex conjugate transpose, and pseudoinverse of a matrix or vector, respectively. $[\mathbf{H}]_{(1,:)}$ denotes the operation to select the first row vector of the matrix H. $\operatorname{diag}\{\mathbf{x}\}$ denotes a square diagonal matrix whose diagonal elements are entries of vector $\mathbf{x} . \max \{a, b\}$ returns the maximum value between $a$ and $b . \min \{a, b\}$ returns the minimum value between $a$ and $b . Q(x)=(1 / \sqrt{2 \pi}) \int_{x}^{\infty} e^{-\left(\omega^{2} / 2\right)} d \omega$ denotes the $Q$ function.

\section{SySTEM MODEL}

We consider an FD AF relay-assisted OFDM system in the uplink depicted in Fig. 1. The source is equipped with a single antenna. The AF relay has $N_{t}$ transmit antennas and $N_{m}$ receive antennas, $N_{t}=N_{m}$. The destination is equipped with $N_{d}$ receive antennas. In the FD mode, the relay transmits 


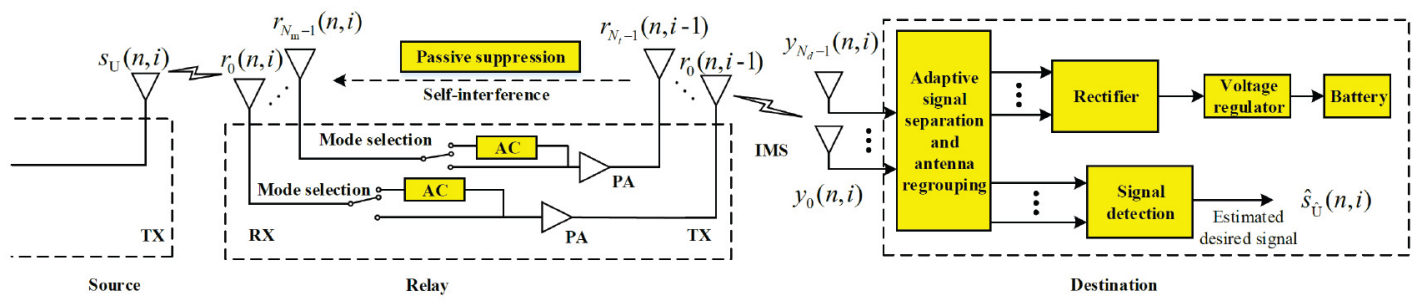

Fig. 1. Block diagram of FD AF relay-assisted wireless systems. (TX: transmitter, RX: receiver, IMS: information of mode selection).

and receives signals at same time and same frequency, which causes SI. We apply an adaptive SI processing mode selection to maintain the SI energy at a reasonable level. The details of the adaptive SI processing mode selection strategy are presented in Section III. The information of mode selection (IMS) is transmitted with the transmitted signal from relay to destination, to assist ICA-based adaptive signal separation or antenna regrouping. In order to measure the residual SI after SI cancelation, we define $\beta$ as the ratio of the SI power before and after suppression/cancelation. $\alpha_{\mathrm{SR}}$ and $\alpha_{\mathrm{RD}}$ denote the path loss from source to relay and the path loss from relay to destination, respectively. All the channels are modeled as the Rayleigh frequency-selective fading channel [29], [30], where the channel of $L$ paths, remains constant for a frame duration of $N_{s}$ OFDM blocks with $N$ subcarriers. Each OFDM block is prepended with a cyclic prefix (CP) of length $L_{\mathrm{cp}}\left(L_{\mathrm{cp}} \geq L-1\right)$ before transmission, which is removed at destination to avoid interblock interference.

Let $s_{U}(n, i)$ denote the transmitted quadrature phase-shift keying (QPSK) symbol on the $n$th $(n=0,1, \ldots, N-1)$ subcarrier in the $i$ th $\left(i=0,1, \ldots, N_{s}-1\right)$ OFDM block. A nonredundant precoding mechanism is expressed via superposition of source data and reference data as follows [28]:

$$
s_{U}(n, i)=\frac{1}{\sqrt{1+a^{2}}}\left[d(n, i)+a d_{\mathrm{ref}}(n, i)\right]
$$

where $d_{\mathrm{ref}}(n, i)$ is the reference symbol used at destination to eliminate the ambiguity caused by ICA. $d(n, i)$ is the source symbol, and $a(0 \leq a \leq 1)$ is the precoding constant which makes a tradeoff in power allocation between the source symbol and the reference symbol.

Let $H_{m}^{[\mathrm{SR}]}(n)$ and $H_{m, t}^{[\mathrm{RR}]}(n)$ denote the channel frequency response matrices on the $n$th subcarrier, between the user and the $m$ th $\left(m=0,1, \ldots, N_{m}-1\right)$ receive antenna at relay, and between the $m$ th receive antenna and the $t$-th $\left(t=0,1, \ldots, N_{t}-1\right)$ transmit antenna at relay, respectively. The received signal in the frequency domain on the $n$th subcarrier at the $m$ th receive antenna of the relay is given by

$$
\begin{aligned}
r_{m}(n, i)= & \underbrace{\sqrt{P_{s} \alpha_{\mathrm{SR}}} H_{m}^{[\mathrm{SR}]}(n) s_{U}(n, i)}_{\text {Desired Signal }} \\
& +\underbrace{\sqrt{\frac{1}{\beta} \sum_{t=0}^{N_{t}-1} H_{m, t}^{[\mathrm{RR}]}(n) r_{t}(n, i-\tau)}+z_{m}(n, i)}_{\text {Residual SI }}
\end{aligned}
$$

where $P_{s}$ is the transmitted power at source, and $z_{m}(n, i)$ is the additive white Gaussian noise (AWGN) with zero mean and variance of $N_{0}$. The transmitted signal at relay is a delayed version of $\tau \geq 1$ symbols due to the relay processing delay. The transmitted signal on the $n$th subcarrier at the $t$-th transmit antenna of the relay is given by

$$
r_{t}(n, i)= \begin{cases}0, & \text { for } 0 \leq i \leq \tau-1 \\ \sqrt{\beta_{\mathrm{PA}}} r_{m}(n, i), & \text { for } i \geq \tau\end{cases}
$$

where $\beta_{\mathrm{PA}}$ denotes power amplifier (PA) gain at relay, given by

$$
\beta_{\mathrm{PA}}=\frac{P_{r}}{\sum_{n=1}^{N}\left|H^{[\mathrm{SR}]}(n)\right|^{2} \alpha_{\mathrm{SR}} P_{s}+N_{0}} .
$$

Without loss of generality, we assume that the SI cancelation at FD relay takes a processing delay of one symbol period [31], i.e., $\tau=1$. For $i \geq 1$, by recursively implementing (2) and (3), the transmit signal at relay can be re-expressed as

$$
\begin{aligned}
r_{t}(n, i)= & \sqrt{\beta_{\mathrm{PA}} P_{s} \alpha_{\mathrm{SR}}} H_{m}^{[\mathrm{SR}]}(n) s_{U}(n, i)+\sqrt{\beta_{\mathrm{PA}} F} z_{m}(n, i) \\
& +\sqrt{\beta_{\mathrm{PA}}} \sum_{j=1}^{\infty}\left(\sqrt{\frac{\beta_{\mathrm{PA}}}{\beta}} \sum_{t=0}^{N_{t}-1} H_{m, t}^{[\mathrm{RR}]}(n)\right)^{j} \\
& \times\left[\sqrt{P_{s} \alpha_{\mathrm{SR}}} H_{t}^{[\mathrm{SR}]}(n) s_{U}(n, i-j)+\sqrt{F} z_{t}(n, i-j)\right]
\end{aligned}
$$

$$
\begin{aligned}
y_{d}(n, i)= & \sum_{t_{1}=0}^{N_{t}-1} \sqrt{\beta_{\mathrm{PA}} P_{s} \alpha_{\mathrm{SR}} \alpha_{\mathrm{RD}}} H_{d, t_{1}}^{[\mathrm{RD}]}(n) H_{t_{1}}^{[\mathrm{SR}]}(n) s_{U}(n, i) \\
& +\sum_{t_{1}=0}^{N_{t}-1} \sqrt{\beta_{\mathrm{PA}} \alpha_{\mathrm{RD}}} H_{d, t_{1}}^{[\mathrm{RD}]}(n) \sum_{j=1}^{\infty}\left(\sqrt{\left.\frac{\beta_{\mathrm{PA}}}{\beta} \sum_{t=0}^{N_{t}-1} H_{t_{1}, t}^{[\mathrm{RR}]}(n)\right)^{j} \sqrt{P_{s} \alpha_{\mathrm{SR}}} H_{t}^{[\mathrm{SR}]}(n) s_{U}(n, i-j)}\right. \\
& +\sum_{t_{1}=0}^{N_{t}-1} \sqrt{\beta_{\mathrm{PA}} \alpha_{\mathrm{RD}}} H_{d, t_{1}}^{[\mathrm{RD}]}(n)\left[\sum_{j=1}^{\infty}\left(\sqrt{\frac{\beta_{\mathrm{PA} F}}{\beta}} \sum_{t=0}^{N_{t}-1} H_{t_{1}, t}^{[\mathrm{RR}]}(n)\right)^{j} z_{t}(n, i-j)+\sqrt{F} z_{t_{1}}(n, i)\right]+z_{d}(n, i)
\end{aligned}
$$


$r_{m}(n, i)$ is the signal to be amplified by PA, and retransmitted to the destination. The received signal $y_{d}(n, i)$ on the $n$th subcarrier at the $d$ th $\left(d=0,1, \ldots, N_{d}-1\right)$ antenna of the destination is written as

$$
y_{d}(n, i)=\sum_{t=0}^{N_{t}-1} \sqrt{\alpha_{\mathrm{RD}}} H_{d, t}^{[\mathrm{RD}]}(n) r_{t}(n, i)+z_{d}(n, i)
$$

where $H_{d, t}^{[\mathrm{RD}]}(n)$ is the channel frequency response from the $t$-th transmit antenna at relay to the $d$ th receiver antenna at destination, and $z_{d}(n, i)$ is the noise. Substituting (5) into (6) yields the received signal at destination, as expressed in (7) shown at the bottom of the previous page.

Define $s_{I}(n, i)$ as the SI symbol on the $n$th subcarrier in the $i$ th block, and $H_{I, d}(n)$ as the equivalent SI channel frequency response on the $d$ th receive antenna at destination denoting the channel frequency response on the $n$th subcarrier. At destination, the $\mathrm{CP}$ is first removed. Then, the received signals are transformed to the frequency domain. $\tilde{y}_{d}(n, i)$ is defined as the received symbol on the $n$th subcarrier in the $i$ th block, which is given by

$$
\tilde{y}_{d}(n, i)=\underbrace{H_{U, d}(n) s_{U}(n, i)}_{\text {Desired Signal }}+\underbrace{H_{I, d}(n) s_{I}(n, i)}_{\text {Residual SI }}+\underbrace{\tilde{z}_{d}(n, i)}_{\text {Equivalent Noise }}
$$

where $s_{I}(n, i)=s_{U}(n, i-1)$ is the SI, and

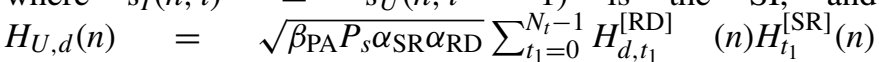
is the equivalent channel frequency response of the desired signal on the $d$ th antenna at destination. $H_{I, d}(n)=\sqrt{\left[\left(\beta_{\mathrm{PA}}^{2} P_{s} \alpha_{\mathrm{SR}} \alpha_{\mathrm{RD}}\right) / \beta\right]} \sum_{t_{1}=0}^{N_{t}-1} \sum_{t=0}^{N_{t}-1}$ $H_{d, t_{1}}^{[\mathrm{RD}]}(n) H_{t_{1}, t}^{[\mathrm{RR}]}(n) H_{t}^{[\mathrm{SR}]}(n)$ is the equivalent channel frequency response of SI, and $\tilde{z}_{d}(n, i)$ is expressed as (9), shown at the bottom of the page, which is the equivalent noise.

\section{Adaptive Si Processing Mode Selection}

In this section, we apply an adaptive SI processing mode selection to maintain the SI energy at a reasonable level. There are four SI processing modes described as follows.

1) PSAC-S: SI is canceled by $\mathrm{PS}$ and $\mathrm{AC}$ at relay and the residual $\mathrm{SI}$ is treated as source at destination.

2) $P S A C-N$ : SI is canceled by PS and $\mathrm{AC}$ at relay and the residual $\mathrm{SI}$ is treated as noise at destination.

3) PS-S: SI is canceled by PS only at relay and the residual SI is treated as source at destination.

4) $P S-N$ : SI is canceled by PS at relay and the residual SI is treated as noise at destination. Two thresholds of SRSIR are applied at relay to help determine the SI processing modes.

\section{A. SRSIR at Relay}

Let $q_{m}(n)=\left(\beta_{\mathrm{PA}} / \beta\right) \sum_{t=0}^{N_{t}-1}\left|H_{m, t}^{[\mathrm{RR}]}(n)\right|^{2}$ denote the power coefficient of the loop channel of the SI on the $n$th subcarrier. The SRSIR on the $n$th subcarrier at the $m$ th antenna of the relay can be derived as

$$
\gamma_{R, m}(n)=10 \lg \left(\frac{P_{s} \alpha_{\mathrm{SR}}\left|H_{m}^{[\mathrm{SR}]}(n)\right|^{2}}{\sum_{j=1}^{N_{s}-1}\left(q_{m}(n)\right)^{j} P_{s} \alpha_{\mathrm{SR}}\left|H_{t}^{[\mathrm{SR}]}(n)\right|^{2}}\right) .
$$

Equation (10) can be summarized as

$$
\gamma_{R, m}(n)=10 \lg \left(\frac{1-q_{m}(n)}{q_{m}(n)-\left(q_{m}(n)\right)^{N_{s}-1}}\right)
$$

where $q_{m}(n)^{i}$ can be estimated as

$$
\hat{q}_{m}(n)^{i}=\frac{P_{m}(n, i)-P_{m}(n, i-1)}{P_{m}(n, 1)}
$$

where $P_{m}(n, i)$ is the power of the $i$ th $\left(i=0,1, \ldots, N_{s}-1\right)$ OFDM block on the $n$th subcarrier at the $m$ th receive antenna of the relay. Therefore, the system can realize switching of the SI cancelation mode of PS and PSAC to ensure BER performance and reduce the power consumption of SI cancelation. The SRSIR on the $n$th subcarrier at the $m$ th antenna of the relay can be estimated as

$$
\hat{\gamma}_{R, m}(n)=10 \lg \left(\frac{1}{\sum_{j=1}^{N_{s}-1} \hat{q}_{m}(n)^{j}}\right) .
$$

\section{B. SRSIR Thresholds at Relay for the SI Processing Mode Selection}

The SI processing mode selection depends on the power ratio of the desired signal and the residual SI signal after PS. Let $g_{m}(n)=P_{s} \alpha_{\mathrm{SR}}\left|H_{m}^{[\mathrm{SR}]}(n)\right|^{2}$ denote the power coefficient of the desired signal on the $n$th subcarrier, $P_{R, U}(n, i)=$ $g_{m}(n)\left|s_{U}(n, i)\right|^{2}$ denote the power of the desired signal of current block on the $n$th subcarrier, and $P_{R, I}(n, i)=$ $q_{m}(n) g_{m}(n)\left|s_{U}(n, i-1)\right|^{2}$ denote the power of the current SI signal block on the $n$th subcarrier, $P_{R, I}(n, i)$ and $P_{R, U}(n, i)$ are estimated as

$$
\begin{aligned}
& \hat{P}_{R, I}(n, i)=\left(\left|P_{m}(n, i)-P_{m}(n, i-1)\right|\right) \\
& \hat{P}_{R, U}(n, i)=\left(\left|P_{m}(n, i)-\sum_{k=1}^{i} \hat{P}_{m}(n, k)\right|\right) .
\end{aligned}
$$

When the power of desired signal is equal to or lesser than that of the current SI signal block $P_{R, U}(n, i) \leq P_{R, I}(n, i)$, the SI is canceled by PSAC. When $P_{R, U}(n, i)>P_{R, I}(n, i)$ the SI is suppressed only by PS. Note that the power of the desired signal is normalized $\left|s_{U}(n, 0)\right|^{2}=\left|s_{U}(n, 1)\right|^{2}=\cdots=$

$$
\begin{aligned}
\tilde{z}_{d}(n, i)= & \sqrt{\frac{\beta_{\mathrm{PA}}^{2} \alpha_{\mathrm{RD}}}{\beta} \sum_{t_{1}=0}^{N_{t}-1} \sum_{t=0}^{N_{t}-1} \sum_{t_{2}=0}^{N_{t}-1} H_{d, t_{1}}^{[\mathrm{RD}]}(n) H_{t_{1}, t}^{[\mathrm{RR}]}(n) H_{t, t_{2}}^{[\mathrm{RR}]}(n) r_{t_{2}}(n, i-2)} \\
& +\sqrt{\frac{\beta_{\mathrm{PA}}^{2} F \alpha_{\mathrm{RD}}}{\beta}} \sum_{t_{1}=0}^{N_{t}-1} \sum_{t=0}^{N_{t}-1} H_{d, t_{1}}^{[\mathrm{RD}]}(n) H_{t_{1}, t}^{[\mathrm{RR}]}(n) z_{t}(n, i-1)+\sqrt{\beta_{\mathrm{PA}} F \alpha_{\mathrm{RD}}} \sum_{t_{1}=0}^{N_{t}-1} H_{d, t_{1}}^{[\mathrm{RD}]}(n) z_{t_{1}}(n, i)+z_{d}(n, i)
\end{aligned}
$$




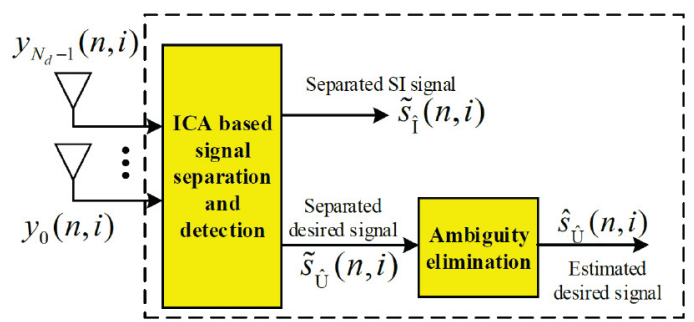

(a)

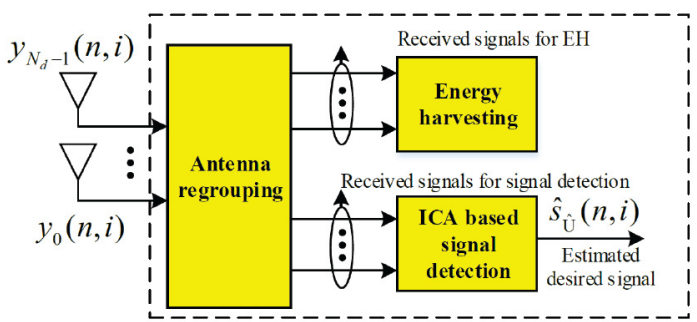

(b)

Fig. 2. Block diagram of the receiver at destination with (a) ICA-based signal separation and detection for PSAC-S and PS-S modes at low SRSIR and (b) SS-based signal separation and ICA-based signal detection for PSAC-N and PS-N modes at high SRSIR.

$\left|s_{U}\left(n, N_{s}-1\right)\right|^{2}=1$. Therefore, a threshold of the SI processing mode selection is applied to determine whether the SI is canceled by PS or PSAC at relay, which can be given as

$$
\gamma_{\mathrm{Th} 1}(n, i)=\gamma_{R}(n)+10 \lg \left(\frac{P_{R, I}(n, i)}{P_{R, U}(n, i)}\right) .
$$

At destination, the ICA-assisted adaptive signal processing algorithm depends on the power of SI at relay. When the power of SI signal is relatively high, the residual SI is regarded as useful signal. When the power of SI signal is relatively low, the residual SI is regarded as noise. When $P_{R, U}(n, i) \leq$ $\sum_{j=1}^{\infty}\left(q_{m}(n)\right)^{j-1} P_{R, I}(n, i-j)$, the residual SI is regarded as useful signal. When $P_{R, U}(n, i)>\sum_{j=1}^{\infty}\left(q_{m}(n)\right)^{j-1} P_{R, I}(n, i-j)$, the residual SI is regarded as noise. Therefore, another higher threshold of the SI processing mode selection is applied to determine whether the residual SI is regarded as useful signal or noise at destination, which can be given as

$$
\gamma_{\mathrm{Th} 2}(n, i)=\gamma_{R}(n)+10 \lg \left(\frac{\sum_{j=1}^{\infty}\left(q_{m}(n)\right)^{j-1} P_{R, I}(n, i-j)}{P_{R, U}(n, i)}\right) .
$$

The conditions for the SI processing mode selection are shown in Table I, where $\gamma_{R}^{[\mathrm{PS}]}$ denotes the SRSIR at relay after PS, and $\gamma_{R}^{[\mathrm{PSAC}]}$ denotes the SRSIR at relay after PSAC. $\gamma_{\mathrm{Th} 1}(n, i)$ and $\gamma_{\mathrm{Th} 2}(n, i)$ are abbreviated as $\gamma_{\mathrm{Th} 1}$ and $\gamma_{\mathrm{Th} 2}$. When SRSIR is less than the lower threshold, the SI is canceled by PSAC, which is suitable for PSAC-S and PSAC-N modes. When SRSIR is larger than the lower threshold, the SI is suppressed only by PS, which is suitable for PS-S and PS-N modes. When SRSIR is between the lower and higher threshold, the residual SI is treated as useful signal to enhance the degree of freedom in signal detection, which is suitable for PSAC-S and PS-S modes. When SRSIR is larger than the higher threshold, the residual SI is treated as a green source for EH, which is suitable for PSAC-N and PS-N modes.

\section{ICA-Assisted Adaptive Signal Separation, DETECTION, AND ENERGY RECYCLING}

We propose an ICA-SS-based adaptive signal detection and energy recycling structure for different SI processing modes. According to the SI processing mode selection, we propose two approaches, as shown in Fig. 2. In the modes of PSAC-S and PS-S, ICA is employed on at least two receive antennas at destination to allow separation of the desired signal
TABLE I

Conditions of Adaptive Mode Selection for Si Processing

\begin{tabular}{cc}
\hline SI processing Mode & Condition \\
\hline PSAC-S & $\gamma_{\mathrm{R}}^{[\mathrm{PS}]}<\gamma_{\mathrm{Th} 1}, \gamma_{\mathrm{Th} 1} \leq \gamma_{\mathrm{R}}^{[\mathrm{PSAC}]}<\gamma_{\mathrm{Th} 2}$ \\
PSAC-N & $\gamma_{\mathrm{R}}^{\mathrm{PS}]}<\gamma_{\mathrm{Th} 1}, \gamma_{\mathrm{R}}^{[\mathrm{PSAC}]} \geq \gamma_{\mathrm{Th} 2}$ \\
PS-S & $\gamma_{\mathrm{Th} 1} \leq \gamma_{\mathrm{R}}^{\mathrm{PS}]}<\gamma_{\mathrm{Th} 2}$ \\
PS-N & $\gamma_{\mathrm{R}}^{[\mathrm{PS}]} \geq \gamma_{\mathrm{Th} 2}$ \\
\hline
\end{tabular}

and SI, and then the desired signal is decoded via ambiguity elimination. In the modes of PSAC-N and PS-N, at least one receive antenna with higher SRSIR is selected for signal detection, while the other antennas are used for energy recycling. This is essentially different from the conventional FD transmission methods [14], [16], where SI is canceled as much as possible. Moreover, the proposed structure provides higher resource utilization over the TSP [18] and PSP [22]-based selfenergy recycling approaches, since the TSP and PSP-based approaches fail to achieve consecutive FD transmission all the time due to the use of partial resources (time or power) for $\mathrm{EH}$.

\section{A. ICA-Based Signal Separation and Detection for Low SRSIR Case}

In the SI processing mode of PSAC-S or PS-S, the SRSIR of the relay is less than the higher threshold $\gamma_{R}<\gamma_{\mathrm{Th} 2}$, ICA is employed on at least two antennas to separate the desired signal and SI. The cross correlation between ICA separated signals and reference signals is explored to detect the desired signals.

ICA is an efficient blind source separation technique by maximizing the non-Gaussianility of received signals. Since ICA requires no training for channel estimation (CE), it is more spectrum efficient than the conventional CE methods [25]. Among different ICA-based methods, the joint approximate diagonalization of eignmatrices (JADEs) [26], a well established batch algorithm based on joint diagonalization of the cumulant matrices of the received components, requires shorter data sequences than other ICA methods. Thus, JADE is employed in this article to perform semi-blind joint signal separation and detection.

Let $\mathbf{s}(n, i)=\left[s_{U}(n, i), s_{I}(n, i)\right]^{\mathrm{T}}$ denote the transmitted signal vector. Let $\mathbf{y}(n, i)=\left[y_{0}(n, i), y_{1}(n, i), \ldots, y_{N_{d}-1}(n, i)\right]^{\mathrm{T}}$ denote all received signals from $N_{d}$ receive antennas of the destination on the $n$th subcarrier in the $i$ th OFDM block, which 
is calculated as

$$
\mathbf{y}(n, i)=\mathbf{H}(n) \mathbf{s}(n, i)+\tilde{\mathbf{z}}(n, i)
$$

where $\mathbf{H}(n)=\left[\mathbf{h}_{U}(n), \mathbf{h}_{I}(n)\right]$ with $\mathbf{h}_{U}(n)=$ $\left[H_{U, 0}(n), H_{U, 1}(n), \ldots, H_{U, N_{d}-1}(n)\right]^{\mathrm{T}} \quad$ and $\quad \mathbf{h}_{I}(n)=$ $\left[H_{I, 0}(n), H_{I, 1}(n), \ldots, H_{I, N_{d}-1}(n)\right]^{\mathrm{T}}, \quad$ and $\tilde{\mathbf{z}}(n, i)=$ $\left[\tilde{z}_{0}(n, i), \tilde{z}_{1}(n, i), \ldots, \tilde{z}_{N_{d}-1}(n, i)\right]^{\mathrm{T}}$. As the received signals $\mathbf{y}(n, i)$ in (18) are a linear mixture of the desired signal $s_{U}(n, i)$ and the SI $s_{I}(n, i)$ on each subcarrier. Thus, JADE is employed on $\mathbf{y}(n, i)$ in (18) to perform separation of desired signal and SI. For the ICA approach, we can obtain the equalized signals as $\tilde{\mathbf{S}}_{\mathrm{LSIR}}(n, i)=\left[\tilde{s}_{\mathrm{LSIR}, U}(n, i), \tilde{s}_{\mathrm{LSIR}, I}(n, i)\right]^{\mathrm{T}}[25]-[27]$, derotated by the phase of each substream as follows [27]:

$$
\check{\mathbf{S}}_{\mathrm{LSIR}}(n, i)=\mathbf{G}(n) \tilde{\mathbf{S}}_{\mathrm{LSIR}}(n, i)
$$

where $\quad \check{\mathrm{s}}_{\mathrm{LSIR}}(n, i)=\left[\check{s}_{\mathrm{LSIR}, U}(n, i), \check{s}_{\mathrm{LSIR}, I}(n, i)\right]^{\mathrm{T}}$, $\mathbf{G}(n)=\operatorname{diag}\left\{\left[g_{U}(n), g_{I}(n)\right]^{\mathrm{T}}\right\}$, with $g_{U}(n)=\alpha_{U}(n) /\left|\alpha_{U}(n)\right|$, $\alpha_{U}(n)=\left\{\left(1 / N_{s}\right) \sum_{i=0}^{N_{s}-1}\left[\tilde{s}_{\mathrm{LSIR}, U}(n, i)\right]^{4}\right\}^{-(1 / 4)} e^{j(\pi / 4)}$ and $g_{I}(n) \quad=\quad \alpha_{I}(n) /\left|\alpha_{I}(n)\right|, \quad \alpha_{I}(n)=$ $\left\{\left(1 / N_{S}\right) \sum_{i=0}^{N_{s}-1}\left[\tilde{s}_{\mathrm{LSIR}, I}(n, i)\right]^{4}\right\}^{-(1 / 4)} e^{j(\pi / 4)} . \quad \alpha_{U}(n) \quad$ and $\alpha_{I}(n)$ denote the factors obtained from $\tilde{s}_{\mathrm{LSIR}, U}(n, i)$ and $\tilde{s}_{\text {LSIR,I }}(n, i)$ for QPSK modulation, respectively, [27].

In the next step, we need to find the desired signal and the SI. Define $\rho_{U}(n)$ and $\rho_{I}(n)$ as the cross-correlations between two equalized signals and the reference signal, respectively, which are given by

$$
\begin{aligned}
\rho_{U}(n) & =\frac{1}{N_{s}} \sum_{i=0}^{N_{s}-1}\left\{\check{s}_{\mathrm{LSIR}, U}(n, i) d_{\mathrm{ref}}^{*}(n, i)\right\} \\
\rho_{I}(n) & =\frac{1}{N_{s}} \sum_{i=0}^{N_{s}-1}\left\{\check{s}_{\mathrm{LSIR}, I}(n, i) d_{\mathrm{ref}}^{*}(n, i)\right\} .
\end{aligned}
$$

By applying permutation ambiguity elimination, the order of the desired signals can be identified by

$$
\hat{U}=\max \left\{\left|\rho_{U}(n)\right|,\left|\rho_{I}(n)\right|\right\} .
$$

By applying quadrant ambiguity elimination [28], the desired signal is given by

$$
\hat{s}_{\text {LSIR, }, \hat{U}}(n, i)=\left[e^{-j \frac{\pi}{4}} \operatorname{sign}\left(\frac{\rho_{\hat{U}}(n)}{\left|\rho_{\hat{U}}(n)\right|} e^{j \frac{\pi}{4}}\right)\right]^{-1} \check{s}_{\operatorname{LSIR}, \hat{U}}(n, i) .
$$

The order of SI can be identified by

$$
\hat{I}=\min \left\{\left|\rho_{U}(n)\right|,\left|\rho_{I}(n)\right|\right\} .
$$

With the identified SI order of $\hat{I}$, the $\operatorname{SI} \check{S}_{\mathrm{LSIR}, \hat{I}}(n, i)$ are extracted from the received signals. The quadrant ambiguity elimination is not required for the SI.

\section{B. SS-Based Signal Separation and ICA-Based Signal Detection for High SRSIR Case}

In the SI processing mode of PSAC-N or PS-N, when the SRSIR of the relay is equal to or larger than the higher threshold $\gamma_{R} \geq \gamma_{\text {Th2 }}$, the SI power is low. There is no need to separate the SI from the received signals. The SI power can be incorporated into the noise. At least one of antennas with highest SIRs can be used for signal detection, while other antennas are utilized for SS-based energy recycling.

From (8), the SRSIR on the $n$th subcarrier of the destination can be derived as

$$
\gamma_{d}=10 \lg \left(\frac{P_{U, d}}{P_{I, d}}\right)
$$

where $P_{U, d}=\sum_{n=0}^{N-1} \sum_{i=0}^{N_{s}-1} P_{U, d}(n, i)$ with $P_{U, d}(n, i)=$ $\left|H_{U, d}(n) s_{U}(n, i)\right|^{2}$ denotes the power of the desired signal on the $d$ th receive antenna at destination, and $P_{I, d}=$ $\sum_{n=0}^{N-1} \sum_{i=0}^{N_{s}-1} P_{I, d}(n, i)$ with $P_{I, d}(n, i)=\left|H_{I, d}(n) s_{I}(n, i)\right|^{2}$ denotes the power of SI on the $d$ th receive antenna at destination.

The transmitted signals are assumed to have constant power. The power difference between a number of consecutive OFDM blocks can be used to estimate the SRSIR, as the SI is from the previous blocks. Thus, QPSK modulation is used in this article. The power of the received signal in the $i$ th block at the $d$ th antenna of the destination is given by

$$
P_{d}(n, i)= \begin{cases}P_{U, d}(n, i)+P_{Z, d}(n, i), & \text { if } i=0 \\ P_{U, d}(n, i)+P_{I, d}(n, i) & \\ +P_{Z, d}(n, i), & \text { if } i=1, \ldots, N_{s}-1\end{cases}
$$

where $P_{Z, d}(n, i)=\left|\tilde{z}_{d}(n, i)\right|^{2}$ is the equivalent noise power. Due to the constant power of the transmitted signals, we have $P_{U, d}(n, 0)=P_{U, d}(n, 1) \cdots=P_{U, d}\left(n, N_{s}-1\right)$. Since the SI is from the previous block, the SI power can be estimated from the power difference between received signals over two consecutive OFDM blocks as

$$
\hat{P}_{I, d}(n, i)=\left(\left|P_{d}(n, i)-P_{d}(n, i-1)\right|\right) .
$$

The power of the desired signal in the $i$ th block is estimated as

$$
\hat{P}_{U, d}(n, i)=\left(\left|P_{d}(n, i)-\sum_{k=1}^{i} \hat{P}_{I, d}(n, k)\right|\right) .
$$

The estimation of SRSIR is given by

$$
\hat{\gamma}_{d}=10 \lg \left(\frac{\hat{P}_{U, d}}{\hat{P}_{I, d}}\right) \text {. }
$$

where $\hat{P}_{U, d}=\sum_{n=0}^{N-1} \sum_{i=0}^{N_{s}-1} \hat{P}_{U, d}(n, i)$ is the estimated power of the desired signal and $\hat{P}_{I, d}=\sum_{n=0}^{N_{-} 1} \sum_{i=0}^{N_{s}-1} \hat{P}_{I, d}(n, i)$ is the estimated power of the SI at the $d$ th antenna.

We select a number of antennas with estimated $N_{q}$ highest SIRs for signal detection, i.e., $\left(\hat{\gamma}_{0}>\hat{\gamma}_{1}, \ldots,>\hat{\gamma}_{N_{q}-1}\right)$, while the rest of $N_{b}$ antennas are used for SS-based energy recycling. Equation (8) can be re-expressed as

$$
y_{d}(n, i)=\underbrace{H_{U, d}(n) s_{U}(n, i)}_{\text {Desired Signal }}+\underbrace{\check{z}_{d}(n, i)}_{\text {Equivalent Noise }}
$$

where $\check{z}_{d}(n, i)=\tilde{z}_{d}(n, i)+H_{I, d}(n) s_{I}(n, i)$ is the equivalent noise including SI. Assume that $N_{q}$ antennas at destination are selected for signal detection by the proposed SS approach. Let $\mathbf{y}_{\mathrm{HSIR}}(n, i)=\left[y_{0}(n, i), y_{1}(n, i), \ldots, y_{N_{q}-1}(n, i)\right]^{\mathrm{T}}$ denote the received signals of $N_{q}$ receive antennas from a total number 
of $N_{d}$ antennas at destination on the $n$th subcarrier in the $i$ th OFDM block, expressed as

$$
\mathbf{y}_{\mathrm{HSIR}}(n, i)=\mathbf{h}_{U}(n) s_{U}(n, i)+\check{\mathbf{z}}(n, i)
$$

where $\mathbf{h}_{U}(n)=\left[H_{U, 0}(n), H_{U, 1}(n), \ldots, H_{U, N_{d}-1}(n)\right]^{\mathrm{T}}$ and $\check{\mathbf{z}}(n, i)=\left[\check{z}_{0}(n, i), \check{z}_{1}(n, i), \ldots, \check{z}_{N_{q}-1}(n, i)\right]^{\mathrm{T}}$. JADE [26] is employed on $\mathbf{y}_{\mathrm{HSIR}}(n, i)$ in (31) for signal detection to obtain the equalized signal $\tilde{s}_{\mathrm{HSIR}}(n, i)$, derotated by the phase of each substream as follows:

$$
\check{s}_{\mathrm{HSIR}}(n, i)=\frac{\alpha(n)}{|\alpha(n)|} \tilde{s}_{\mathrm{HSIR}}(n, i)
$$

where $\quad \alpha(n)=\left\{\left(1 / N_{s}\right) \sum_{i=0}^{N_{s}-1}\left[\tilde{s}_{\mathrm{HSIR}}(n, i)\right]^{4}\right\}^{-(1 / 4)}$ $e^{j(\pi / 4)}$ [27], [28] is obtained from $\tilde{s}_{\mathrm{HSIR}}(n, i)$. The crosscorrelation $\rho_{\operatorname{HSIR}}(n)$ between equalized signal $\check{s}_{\mathrm{HSIR}}(n, i)$ and reference signal $d_{\text {ref }}(n, i)$ on the $n$th subcarrier is defined as

$$
\rho_{\mathrm{HSIR}}(n)=\frac{1}{N_{s}} \sum_{i=0}^{N_{s}-1}\left\{\check{s}_{\mathrm{HSIR}}(n, i) d_{\mathrm{ref}}^{*}(n, i)\right\} .
$$

The remaining quadrant ambiguity is solved by [28]

$$
\hat{s}_{\mathrm{HSIR}}(n, i)=\left[e^{-j \frac{\pi}{4}} \operatorname{sign}\left(\frac{\rho_{\mathrm{HSIR}}(n)}{\left|\rho_{\mathrm{HSIR}}(n)\right|} e^{j \frac{\pi}{4}}\right)\right]^{-1} \check{s}_{\mathrm{HSIR}}(n, i) .
$$

When a number of $N_{q}$ antennas are selected for signal detection, the rest of $N_{b}$ antennas are used for SS-based energy recycling. The recycled power collected from $N_{b}$ antennas can be expressed as

$$
P_{\mathrm{HSIR}, \mathrm{SS}}=\sum_{d=0}^{N_{b}-1} \sum_{i=0}^{N_{s}-1} \sum_{n=0}^{N-1} P_{d}(n, i)
$$

A logistic function-based model is adopted [32], [33], which well describes the saturation effect at high input power level as well as the breakdown effect low input power level. The logistic function-based efficiency can be calculated as

$$
P_{\mathrm{EH}}=\frac{\Psi_{\mathrm{EH}}-P_{\mathrm{sat}} \Omega}{1-\Omega}
$$

where $\Psi_{\mathrm{EH}}=\left(P_{\text {sat }} /\left[1+\exp \left(-b_{1}\left(P_{\text {in }}-b_{2}\right)\right)\right]\right)$ is the conventional logistic function with respect to the input power of the harvester $P_{\text {in. }} . P_{\text {sat }}$ denotes the maximum harvested power when the harvesting circuit is saturated. Parameter $\Omega$ is calculated as $\Omega=\left(1 /\left[1+\exp \left(b_{1} b_{2}\right)\right]\right)$, where $b_{1}$ and $b_{2}$ are parameters accounting for physical hardware phenomena, such as the turn-on voltage of the diode and the maximum output power of the rectifier. The values of $P_{\text {sat }}, b_{1}$, and $b_{2}$ are related to the specific circuit design and can be determined by the curve fitting method. Using the EH model in (36), the overall harvested energy based on SS is given by

$$
P_{\mathrm{HSIR}}=T P_{\mathrm{EH}}\left(P_{\mathrm{HSIR}, \mathrm{SS}}\right) \text {. }
$$

\section{Performance Analysis}

In this section, we present an extensive analysis, including robustness of adaptive SI processing, resource utilization, maximum throughput, and EE of the proposed system. In addition, we provide the effect of ambiguity elimination for BER performance.

\section{A. Robustness Analysis}

The output SINR of ICA is affected by ambiguity which is not straightforward to analyze. As ICA achieves a very close BER performance to zero forcing (ZF) detection as shown in Section VII, we use the SINR by ZF detection as a good approximation to ICA, which is given by

$$
\Gamma=\frac{1}{N} \sum_{n=0}^{N-1} \frac{\mathbf{w}_{n} \mathbf{R}_{y y} \mathbf{w}_{n}^{\mathrm{H}}}{\mathbf{w}_{n} \mathbf{R}_{y y} \mathbf{w}_{n}^{\mathrm{H}}-1}
$$

where $\mathbf{R}_{y y}=\mathbf{H}(n) \mathbf{H}^{\mathrm{H}}(n)+N_{0} \mathbf{I}$. When SI is treated as noise, $\mathbf{w}_{n}$ is the pseudoinverse of $\mathbf{h}_{U}(n)$. According to $\mathbf{h}_{U}^{\mathrm{H}}(n) \mathbf{h}_{U}(n)=$ $\left|\mathbf{h}_{U}(n)\right|^{2}, \mathbf{w}_{n}$ is given by

$$
\mathbf{w}_{n}=\mathbf{h}_{U}^{\mathrm{H}}(n) /\left|\mathbf{h}_{U}(n)\right|^{2} .
$$

When SI is treated as a useful source, $\mathbf{w}_{n}$ is the first row of $\mathbf{H}^{\dagger}(n)$, the pseudoinverse of $\mathbf{H}(n)$, which is given by

$$
\mathbf{w}_{n}=\left[\left(\mathbf{H}^{\mathrm{H}}(n) \mathbf{H}(n)\right)^{-1} \mathbf{H}^{\mathrm{H}}(n)\right]_{(1,:)} .
$$

It is obvious that by treating SI as a useful source, a higher degree of freedom in signal detection can be achieved, especially in the case of low SRSIR.

In order to analyze the robustness of the proposed method against SRSIR, we verify it by analyzing the robustness of the output SINR against the input SRSIR. Let $P_{U}$ and $P_{I}=P_{U} / \gamma_{R}$ denote the power of source signal and SI signal, respectively. We know $\mathbf{H}(n)=\left[\mathbf{h}_{U}(n) \mathbf{h}_{I}(n)\right]$ from (18), $\mathbf{R}_{y y}$ can be expressed as $\mathbf{R}_{y y}=P_{U} \mathbf{h}_{U}(n) \mathbf{h}_{U}^{\mathrm{H}}(n)+\left(P_{U} / \gamma\right) \mathbf{h}_{I}(n) \mathbf{h}_{I}^{\mathrm{H}}(n)+N_{0} \mathbf{I}$. Let $\mathbf{W}_{n}=\mathbf{w}_{n} \mathbf{R}_{y y} \mathbf{w}_{n}^{\mathrm{H}}$. For high SRSIR, SI is treated as noise, $\mathbf{W}_{n}$ is given by

$$
\mathbf{W}_{n}=P_{U} a_{\mathrm{UU}}+\frac{P_{U} a_{\mathrm{UI}}^{2}}{\gamma_{R} a_{\mathrm{UU}}}+N_{0} \mathbf{I}
$$

It can be seen from the above analysis that $\mathbf{W}_{n}$ decreases with the increase of SRSIR and the corresponding output SINR increases. Therefore, in the high SRSIR case, the output SINR is large enough and the system BER performance is considerable.

For low SRSIR, SI is treated as source. $\mathbf{W}_{n}$ is given by

$$
\mathbf{W}_{n}=\frac{P_{U}\left(a_{\mathrm{UU}}^{2} a_{\mathrm{II}}^{2}-2 a_{\mathrm{UI}}^{2} a_{\mathrm{UU}} a_{\mathrm{II}}+a_{\mathrm{UI}}^{4}\right)}{\left(a_{\mathrm{UU}} a_{\mathrm{II}}-a_{\mathrm{UI}}^{2}\right)}+a_{\mathrm{II}} N_{0} \mathbf{I}
$$

where $a_{\mathrm{UU}}=\mathbf{h}_{U}^{\mathrm{H}}(n) \mathbf{h}_{U}(n), a_{\mathrm{II}}=\mathbf{h}_{I}^{\mathrm{H}}(n) \mathbf{h}_{I}(n), a_{\mathrm{UI}}=$ $\mathbf{h}_{U}^{\mathrm{H}}(n) \mathbf{h}_{I}(n)$ and $a_{\mathrm{IU}}=\mathbf{h}_{I}^{\mathrm{H}}(n) \mathbf{h}_{U}(n)$, since we have $a_{\mathrm{UI}}=a_{\mathrm{IU}}$. According to (42), we know $\mathbf{W}_{n}$ is independent of $\gamma_{R}$. From the above analyses, it can be seen that the output SINR is not affected by SRSIR. Therefore, the BER performance of the proposed ICA-SS scheme is robust against SI for low SRSIR.

\section{B. Resource Utilization}

In this section, we present the resource utilization of the proposed ICA-SS scheme, which is defined as the ratio of the number of utilized links to the number of total available links in the scheduling time.

For the HD TSP structure [34], [35], three time slots are required in the scheduling time. There are two links (sourcerelay and relay-destination) available in each time slot (three 
TABLE II

Analyses of Resource Utilization (DM: Duplex Mode, RU: RESOURCE UTILIZATION)

\begin{tabular}{|c|c|c|c|c|}
\hline References & DM & Splitting Structure & SI Processing & RU \\
\hline$[34][35]$ & HD & TSP & $/$ & $1 / 2$ \\
\hline$[36][37]$ & HD & PSP & $/$ & $1 / 2$ \\
\hline$[20]$ & FD & TSP & Cancelation & $1 / 2$ \\
\hline$[18]$ & FD & TSP & Recycling & $2 / 3$ \\
\hline$[21]$ & FD & PSP & Cancelation & $3 / 4$ \\
\hline ICA-SS & FD & ICA/SS & Separation/Recycling & 1 \\
\hline
\end{tabular}

time slots are required), while only one link is actually utilized in each time slot. Therefore, the resource utilization is equal to $(1 \times 3) /(2 \times 3)=1 / 2$.

For the HD PSP [36], [37], two time slots are required and only one link (source-relay or relay-destination) can be utilized in each time slot. Therefore, the resource utilization is equal to $(1 \times 2) /(2 \times 2)=1 / 2$.

For the FD TSP without SI cancelation [20], there are three available links (source-relay or relay-destination and SI link). As mentioned in Section I, in the first time slot, the source sends energy-bearing signal to the relay for EH. In the second time slot, the source sends information-bearing signal to the relay and the relay forwards the received signal to the destination simultaneously. Therefore, only one link is utilized in the first time slot, and the resource utilization is equal to $(1+2) /(3 \times 2)=1 / 2$. For the FD TSP structure with SI recycling [18], since SI link is utilized for recycling energy in the second time slot, the resource utilization is equal to $(1+3) /(3 \times 2)=2 / 3$.

At last, for the FD PSP [21], the relay works in the FD mode and continuously splits part of the received signal for $\mathrm{EH}$, and forwards the remnant signal to the destination for decoding. Since source-destination link is assumed available in the system, the total links become into four (source-relay, relay-destination, source-destination, and SI link). However, SI is canceled as noise and SI link is not utilized. The resource utilization is equal to $(3 \times 2) /(4 \times 2)=3 / 4$.

In summary, existing FD TSP/PSP relay systems can not achieve full resource utilization. In contrast, our proposed scheme achieves full resource utilization via consecutive FD data transmission at all time and the same frequency. The comparisons various systems in terms of resource utilization are presented in Table II.

\section{EE and Throughput Analysis}

In this section, we analyze the EE $\eta$ of the proposed ICA-SS scheme, which is defined as

$$
\eta=\frac{C}{P} .
$$

Since EE involves with throughput $C$ and power consumption $P$, we first give the total power consumption and then demonstrate the maximum system throughput.

The power consumption of the whole system is given by

$$
P=P_{\mathrm{A}}+P_{\mathrm{C}}+P_{\mathrm{PSAC}}+P_{\mathrm{DSP}}-P_{\mathrm{EH}}
$$

where $P_{\mathrm{A}}, P_{\mathrm{C}}, P_{\mathrm{PSAC}}$, and $P_{\mathrm{DSP}}$ indicate $\mathrm{PA}$ power, circuit power, power consumption in the PSAC mode, and digital signal processing (DSP) power, respectively. Let $\eta_{\mathrm{PAE}}$ denote the power added efficiency (PAE) of PA, which describes how much percentage of direct current power is transformed to output power, and is expressed as $\eta_{\mathrm{PAE}}=\left[\left(P_{\text {out }}-P_{\text {in }}\right) / P_{\mathrm{A}}\right]$ [38], where $P_{\text {out }}$ is the output power of PA. Hence, the power consumption of PA can be expressed as [38], [39]

$$
P_{\mathrm{A}}=\frac{P_{s} \alpha_{\mathrm{SR}}\left(\beta_{\mathrm{PA}}-1\right)}{\eta_{\mathrm{PAE}}} \sum_{n=1}^{N}\left|H^{[\mathrm{SR}]}(n)\right|^{2} .
$$

The total circuit power $P_{\mathrm{C}}$ includes static circuit power and dynamic circuit power [14]. The conventional methods [16] require additional AC power $P_{\mathrm{AC}}$ and DC power $P_{\mathrm{DC}}$ to mitigate $\mathrm{SI}$ as much as possible, by a direct-conversion radio architecture [16], while the proposed ICA-assisted scheme implemented by a DSP chip has a power consumption as small as the previous methods (e.g., ZF algorithm [40]). The existing methods [16] require a total power consumption of $P=P_{\mathrm{A}}+P_{\mathrm{C}}+P_{\mathrm{AC}}+P_{\mathrm{DC}}+P_{\mathrm{DSP}}$. Thus, the proposed method provides a power gain of $\sigma_{P}=P_{\mathrm{AC}}+P_{\mathrm{DC}}+P_{\mathrm{EH}}-P_{\mathrm{PSAC}}$ over the conventional methods. Since the proposed scheme cancels SI via PS and/or AC, the power consumption of PSAC tends to be equal to or smaller than that of $\mathrm{AC}$ alone (i.e., $P_{\mathrm{PSAC}} \leq P_{\mathrm{AC}}$ ).

With QPSK modulation, the maximum system throughput $C$ is defined as [41]

$$
C=B \log _{2}\left(1+\frac{-1.5 \Gamma}{\ln (5 \lambda)}\right)
$$

where $B$ denotes bandwidth, $\lambda$ is the target BER, and $\Gamma$ is the SINR of desired signal at destination, which is given by (38).

For the FD TSP-based self-energy recycling scheme [18], in the first time slot, the source sends information-bearing signal to the relay. In the second time slot, the source sends energybearing signal to the relay and SI is recycled at relay, and the relay forwards the received information-bearing signal to the destination simultaneously. Let $C_{1}$ denote the throughput in the first slot. It can be calculated by (38), (39), and (46), except that $\mathbf{H}(n)$ in $\mathbf{R}_{y y}$ in (38) and $\mathbf{h}_{U}(n)$ in (39) are both replaced by $\mathbf{h}_{\mathrm{SR}}(n)$, where $\mathbf{h}_{\mathrm{SR}}(n)$ denotes the equivalent channel frequency response of the desired signal at relay. Similarly, the throughput in the second slot, denoted by $C_{2}$, can be calculated except that $\mathbf{H}(n)$ in $\mathbf{R}_{y y}$ in (38) is replaced by $\mathbf{h}_{U}(n)$. The overall throughput is given by

$$
C_{T}=\min \left\{\omega_{T} C_{1},\left(1-\omega_{T}\right) C_{2}\right\}
$$

where $\omega_{T}\left(0<\omega_{T}<1\right)$ denotes a TSP coefficient.

For the FD PSP-based self-energy recycling scheme [22], the relay in the FD mode splits the received signal into two parts in power domain, of which one is utilized for EH and the other is forwarded to the destination for decoding. Thus, the throughput is calculated by (38), (39), and (46), except that a PSP coefficient $\omega_{\mathrm{P}}\left(0<\omega_{\mathrm{P}}<1\right)$ is multiplied with $\Gamma$ in (46).

\section{BER Due to Ambiguity}

The precoding constant $a$, which gives a tradeoff on the power allocation between the source data and the reference data, plays an important role in the precoding process [28]. 
However, SI also affects the quadrant and permutation ambiguity, where SI cancelation amount $\beta$ is a key factor. With a large valued $\beta$, the influence of SI is insignificant on ambiguity elimination. On the other hand, if $\beta$ is too small and the influence of SI is significant on ambiguity elimination, SI can be treated as source.

In order to formulate the effects of SI cancelation amount, we analyze the effect of the equivalent output noise $\tilde{z}_{d}(n, i)$ in (30) of the ICA equalizer on the quadrant and permutation error probabilities. It can be derived that the BER due to quadrant ambiguity is expressed as

$$
p_{U, \mathrm{quad}}=Q\left(\sqrt{\frac{a^{2} N_{s}}{1+\left(1+a^{2}\right) P_{Z}}}\right)
$$

where $Q(\cdot)$ denotes the $Q$ function, defined as $Q(x)=$ $(1 / \sqrt{2 \pi}) \int_{x}^{\infty} e^{-\left(\omega^{2} / 2\right)} d \omega$. The BER due to the permutation ambiguity can be expressed as

$$
p_{U, \text { perm }}=2 Q\left(\sqrt{\frac{\sqrt{2} a^{2} N_{s}}{1+P_{Z}}}\right) .
$$

High SRSIR Case: If the estimated SRSIR $\gamma_{R} \geq \gamma_{\mathrm{Th} 2}$, the residual SI can be incorporated into noise. In this case, the $P_{Z}$ in (48) and (49) is replaced by

$$
P_{Z, \mathrm{HSIR}}=\sum_{n=0}^{N-1} \sum_{i=0}^{N_{s}-1} \sum_{d=0}^{N_{d}-1}\left[\frac{\beta_{\mathrm{PA}}}{\beta} P_{I, d}(n, i-1)+P_{Z, d}(n, i)\right] .
$$

Low SRSIR Case: If the estimated SRSIR $\gamma_{R}<\gamma_{\mathrm{Th} 2}$, the $i$ th block of SI is treated as useful signal, and the previous $(i-1)$ blocks of SI can be incorporated into noise. In this case, the $P_{Z}$ in (48) and (49) is replaced by

$$
\begin{aligned}
P_{Z, \mathrm{LSIR}}=\sum_{n=0}^{N-1} \sum_{i=0}^{N_{s}-1} \sum_{d=0}^{N_{d}-1}[ & \frac{\beta_{\mathrm{PA}}^{2}}{\beta^{2}} P_{I, d}(n, i-2)+\frac{\beta_{\mathrm{PA}}}{\beta} P_{Z, d} \\
& \left.\times(n, i-1)+P_{Z, d}(n, i)\right] .
\end{aligned}
$$

In order to analyze the asymptotic performance of the ambiguity elimination, we define the BER ratio of the quadrant ambiguity and permutation ambiguity $R_{p q}=$ $\left(p_{U, \text { quad }} / p_{U, \text { perm }}\right)$. Since $Q$ function can be approximated as $Q(x) \cong\left(\left[\exp \left(-x^{2} / 2\right)\right] /[\sqrt{2 \pi} x]\right)(x \gg 1)$, the BER ratio is given by

$$
R_{p q}=2^{-\frac{3}{4}} \frac{1}{\sqrt{q}} \exp \left(\frac{(\sqrt{2}-q) a^{2} N_{s}}{2\left(1+P_{Z}\right)}\right)
$$

where $q=\left[\left(1+P_{Z}\right) /\left(1+P_{Z}+a^{2} P_{Z}\right)\right]$. As the precoding constant $a$ is in the range of $0 \leq a \leq 1$, it can be derived that $(1 / 2)<q \leq 1$. Since $R_{p q}$ is a monotonically decreasing function of variable $q$, the range of the BER ratio can be derived as

$$
R_{p q} \in\left[0.59 \exp \left(\frac{0.21 a^{2} N_{s}}{1+P_{Z}}\right), 0.84 \exp \left(\frac{0.46 a^{2} N_{s}}{1+P_{Z}}\right)\right) .
$$

TABLE III

ANALytical COMPUTATIONAL COMPLEXITy $\left(N_{S}\right.$ : Number OF Blocks, $N$ : Number of SubCarRiers, $N_{d}$ : Number of ReCEIVE ANTENNAS AT Destination, EQ: EQUalization, AE: AMBiguity Elimination)

\begin{tabular}{|l|c|c|}
\hline \multicolumn{2}{|c|}{ Item } & Order of Complexity \\
\hline \multicolumn{2}{|c|}{ Precoding } & $N N_{s}$ \\
\hline \multirow{3}{*}{$\begin{array}{l}\text { ICA EQ and } \\
\text { SI as source }\end{array}$} & ICA (JADE) & $N\left(N_{d}^{2}+16 N_{s}+32\right)$ \\
\cline { 2 - 3 } & Phase AE & $2 N N_{s}$ \\
\cline { 2 - 3 } & Permutation AE & $N N_{s} / 2$ \\
\cline { 2 - 3 } & Phase Rotation & $N N_{s}$ \\
\hline \multirow{2}{*}{ ICA EQ and } & ICA (JADE) & $N\left(N_{d}^{2}+N_{s}\right)$ \\
\cline { 2 - 3 } SS EH & Phase AE & $N N_{s}$ \\
\cline { 2 - 3 } & Phase Rotation & $N N_{s}$ \\
\hline
\end{tabular}

For the high SNR case, $P_{Z}$ tends to zero, and the BER ratio is larger than $0.59 e^{0.21 a^{2} N_{s}}$. If $a$ and $N_{s}$ are set to as 0.26 and 256, respectively, the value of the BER ratio is near by 22. This is also consistent with the simulation results in Section VII. Based on the above analyses, the BER due to quadrant ambiguity plays a dominant role in the performance.

\section{COMPLEXITy ANALYSIS}

In Table III, we present the computational complexity of the proposed ICA-SS, in terms of the number of complex multiplications. Since the reference data in precoding can be designed offline and known in advance at destination. The precoding process, including a superimposition of reference data and source data, requires a complexity of $\mathcal{O}\left(N N_{S}\right)$. The cross-correlation coefficients between equalized signals and reference signals are required to search for the order of the desired signal and SI signal. Thus, its complexity is $\mathcal{O}\left(N N_{s} / 2\right)$. The phase shifting in (19) introduces a new phase rotation which needs to be solved on each equalized symbol. The complexity of $\mathcal{O}\left(N N_{s}\right)$ can be obtained. The computational complexity of the proposed ICA algorithm includes the computational complexity of precoding, ICA equalization (EQ), and SI as source. Thus, its complexity is $\mathcal{O}_{\text {ICA }}=$ $\mathcal{O}\left(4 N N_{s}+N\left(N_{d}^{2}+16 N_{s}+32\right)+N N_{s} / 2\right)$. The computational complexity of the proposed SS algorithm includes the computational complexity of precoding, ICA EQ, and SS EH. Thus, its complexity is $\mathcal{O}_{\mathrm{SS}}\left(3 N N_{s}+N\left(N_{d}^{2}+N_{s}\right)\right)$. The computational complexity of the proposed ICA-SS algorithm is between ICA and SS. Thus, its complexity is $\mathcal{O}_{\mathrm{SS}}\left(3 N N_{s}+N\left(N_{d}^{2}+N_{s}\right)\right) \leq$ $\mathcal{O}_{\text {ICA-SS }} \leq \mathcal{O}_{\text {ICA }}\left(4 N N_{s}+N\left(N_{d}^{2}+16 N_{s}+32\right)+N N_{s} / 2\right)$.

\section{NUMERICAL RESUlTS}

The simulation results are provided to demonstrate the performance of the proposed ICA-SS-assisted FD relay system. System parameters are set as follows: the source and destination are equipped with a single transmit antenna and $N_{d}=2$ receive antennas, respectively; the relay is equipped with $N_{t}=2$ transmit and $N_{m}=2$ receiver antennas; the CSI remains constant during a data frame with $N_{s}=256$ OFDM blocks each with $N=64$ subcarriers; the QPSK modulation scheme is utilized; the channel follows an exponential delay profile with a normalized root mean-square (RMS) delay spread of 1.4; a CP of length $L_{\mathrm{cp}}=16$ is used; the bandwidth is set as $100 \mathrm{MHz}$; the precoding constant is set as $a=0.26$; 


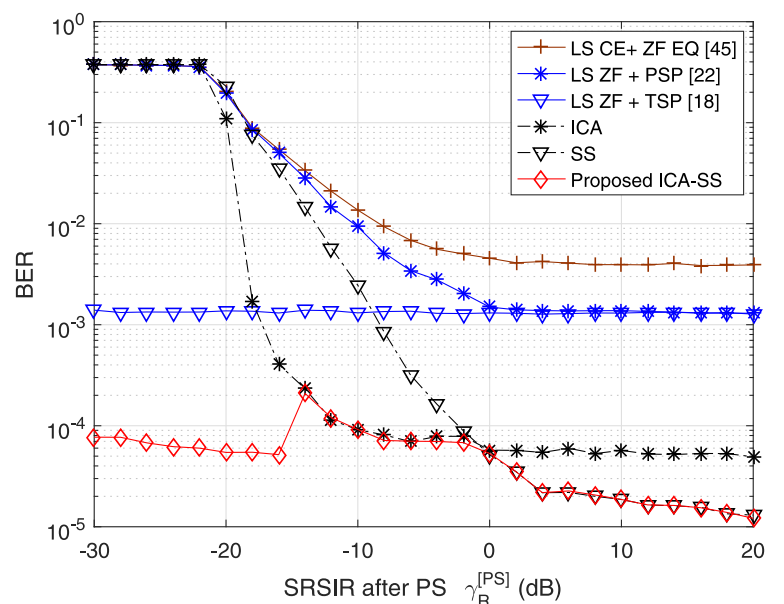

Fig. 3. BER performance of the proposed ICA-SS scheme, with SNR = $20 \mathrm{~dB}$ (CE: channel estimation, EQ: equalization).

the target $\mathrm{BER}=10^{-5}$ is used for EE analysis; the PAE is set to $\eta_{P A E}=40 \%$ [39]; the PA gain is $\beta_{\mathrm{PA}}=36 \mathrm{~dB}$ [42]; the noise figure of each LNA is $F=1.5 \mathrm{~dB}$ [43]; the TSP and PSP coefficients $\omega_{T}$ and $\omega_{\mathrm{P}}$ of the existing methods [18], [22] are both set as 0.5 ; regarding the nonlinear EH model parameters, we assume $P_{\text {sat }}=300 \mathrm{~mW}, b_{1}=10$, and $b_{2}=0.14$ [32]. All the simulation results are averaged over Monte-Carlo runs with independent source data, noise, and channel realizations. ICA and ZF can be implemented by the TMS320VC33 of the DSP processor consuming a power of $P_{\mathrm{DSP}}=200 \mathrm{~mW}$ [44]. The EH model adopts a logistic function-based nonlinear model [32], [33].

Fig. 3 shows the BER performance of the proposed ICA$\mathrm{SS}$ assisted FD relay system in comparison to the existing methods [18], [22], [45] at SNR $=20 \mathrm{~dB}$. The proposed ICA-SS-based signal separation scheme achieves a much better BER performance than the least-square (LS) CE and ZF EQ (LS CE+ZF EQ) [45], the PSP-assisted LS ZF [22] and the TSP-assisted LS ZF [18], especially in the low SRSIR range of $-30 \sim-15 \mathrm{~dB}$. This is due to the adaptive SI processing mode selection, where the residual SI is treated as useful signal to enhance the degree of freedom in signal detection in PSAC-S and PS-S modes, and is treated as noise to enhance the sensitivity of semi-blind signal detection in PSAC-N and PS-N modes. When $\gamma_{R} \leq-2 \mathrm{~dB}$, the ICA-based signal detection method can obtain better BER performance than the SS method. However, when $\gamma_{R} \geq 0 \mathrm{~dB}$, the BER performance of the ICA-based signal separation method is worse than that of the SS method. This indicates the ICA-based signal separation method is more suitable for low SRSIR and the SS-based method is more suitable for high SRSIR. Thus, ICA-SS-based signal separation scheme is proposed to adapt to both low SRSIR and high SRSIR cases. Since the proposed scheme achieves the adaptive SI processing mode selection to balance utilizing SI and canceling SI, it is shown to be more robust against SRSIR than the existing methods [18], [22], [45]. It is noteworthy that the proposed scheme has a small fluctuation from SRSIR $=-16 \mathrm{~dB}$ to SRSIR $=-14 \mathrm{~dB}$ owing to the adaptive mode switching between PSAC and PS.

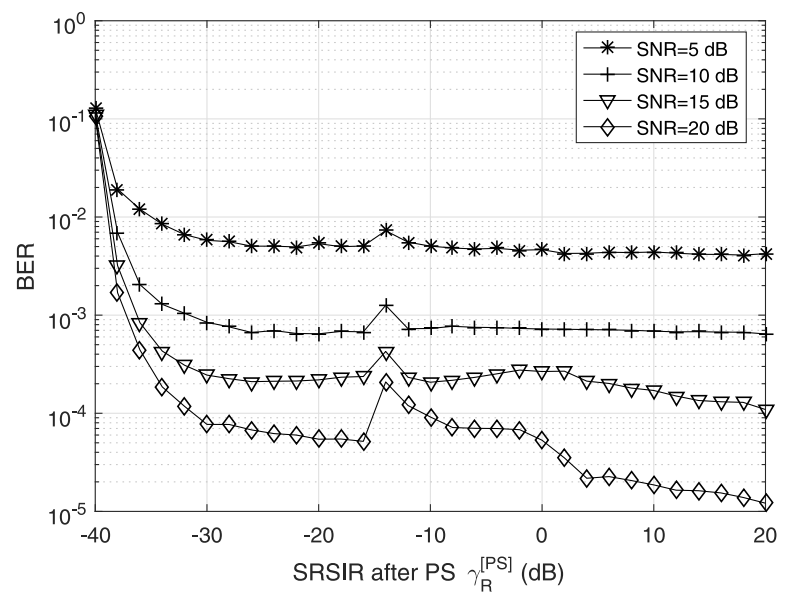

Fig. 4. BER performance of the proposed ICA-SS scheme, with different SNR.

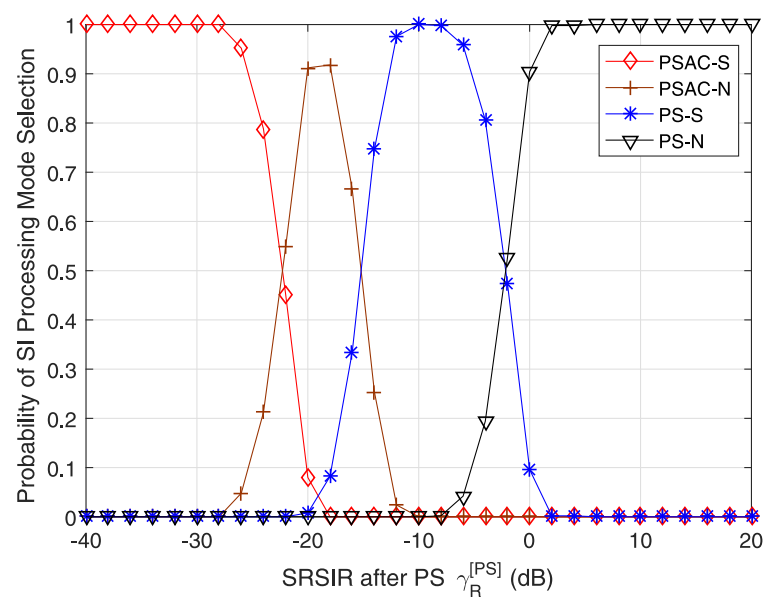

Fig. 5. Probability of the SI processing mode selection, with $\mathrm{SNR}=20 \mathrm{~dB}$.

Fig. 4 demonstrates the BER performance of the proposed ICA-SS-based signal separation scheme with different SNR. They have an inflection point at $-15 \mathrm{~dB}$, which is due to the switch between PSAC and PS modes. In addition, as the increase of SNR, the BER performance of the proposed method is improved accordingly. BER of the proposed ICA-SS scheme is close to $10^{-5}$ at $\gamma_{R}=20 \mathrm{~dB}$.

Fig. 5 shows the probability of SI processing mode selection for $\mathrm{SNR}=20 \mathrm{~dB}$. The probability of using PSAC mode is much higher than that of the PS mode for $\gamma_{R}^{[\mathrm{PS}]}<-15 \mathrm{~dB}$. Conversely, the probability of using PS mode is much higher than that of the PSAC mode for $\gamma_{R}^{[\mathrm{PS}]}>15 \mathrm{~dB}$. This is determined by the first threshold of the SRSIR $\gamma_{\mathrm{Th} 1}$ at relay. The probability of using the PSAC-S mode is the largest for $\gamma_{R}^{[\mathrm{PS}]}<-23 \mathrm{~dB}$ and the probability of using the PS-S mode is the largest for $-15 \mathrm{~dB}<\gamma_{R}^{[\mathrm{PS}]}<-3 \mathrm{~dB}$. The probability of using the PSAC-N mode is the largest for $\gamma_{R}^{[\mathrm{PS}]}>-3 \mathrm{~dB}$. This is determined by the higher threshold of the SRSIR $\gamma_{\mathrm{Th} 2}$ at relay.

Fig. 6 demonstrates the BER performance of the proposed ICA-SS-based signal separation scheme in comparison to the existing methods [18], [22], [45]. Four pilots are required for the existing methods, resulting in much higher training 


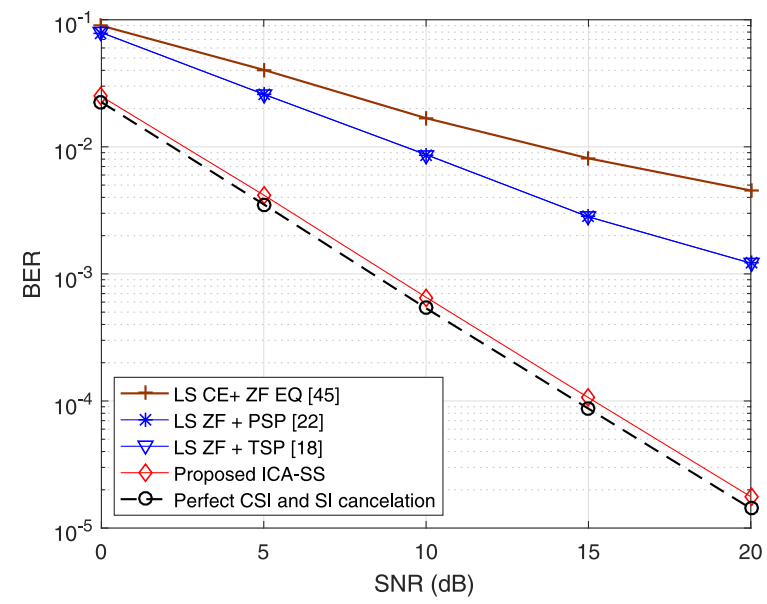

Fig. 6. BER performance of the proposed ICA-SS scheme, with SRSIR after PS $\gamma_{R}^{[\mathrm{PS}]}=20 \mathrm{~dB}$ (CE: channel estimation, EQ: equalization).

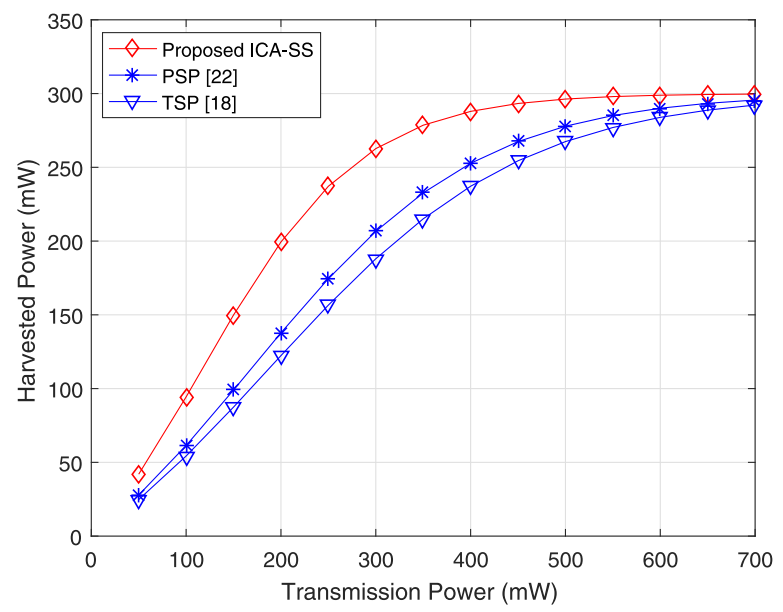

Fig. 7. Harvested power by the proposed ICA-SS approach, compared to that of TSP [18] and PSP [22]-based self-energy recycling schemes.

overhead than the proposed scheme which does not require any side information. As the proposed ICA-SS-based method can make a selection between ICA and SS methods by the adaptive SI processing mode selection, the proposed scheme outperforms the existing methods [18], [22], [45] significantly. For example, at $\mathrm{SNR}=20 \mathrm{~dB}$, the proposed scheme has a performance gain of $16 \mathrm{~dB}$ over LS CE+ZF EQ [45], and of $12 \mathrm{~dB}$ over both PSP-assisted LS ZF [22] and TSP-assisted LS ZF [18]. Also, the proposed scheme provides a performance close to the ideal case with perfect CSI and SI cancelation.

Fig. 7 demonstrates the harvested power of the proposed ICA-SS method with nonlinear EH model, in comparison to the existing methods [18], [22]. The proposed ICA-SS methods harvest more power than TSP [18] and PSP [22]-based self-energy recycling methods. There are about $75 \mathrm{~mW}$ and $65 \mathrm{~mW}$ power gaps between the proposed ICA method and TSP [18] and PSP [22]-based self-energy recycling methods, respectively, with the transmission power at $300 \mathrm{~mW}$. This is due to the fact that the proposed ICA-SS method can achieve full-time and full-power WIPT. On the contrary, only part of

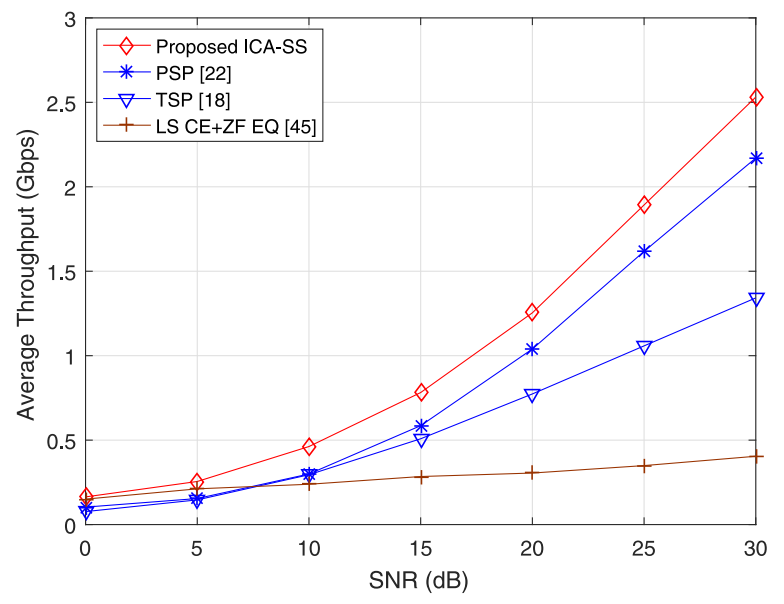

Fig. 8. Throughput of the proposed ICA-SS scheme, in comparison to the existing TSP [18], PSP [22], and LS CE+ZF EQ [45] methods (CE: channel estimation, EQ: equalization).

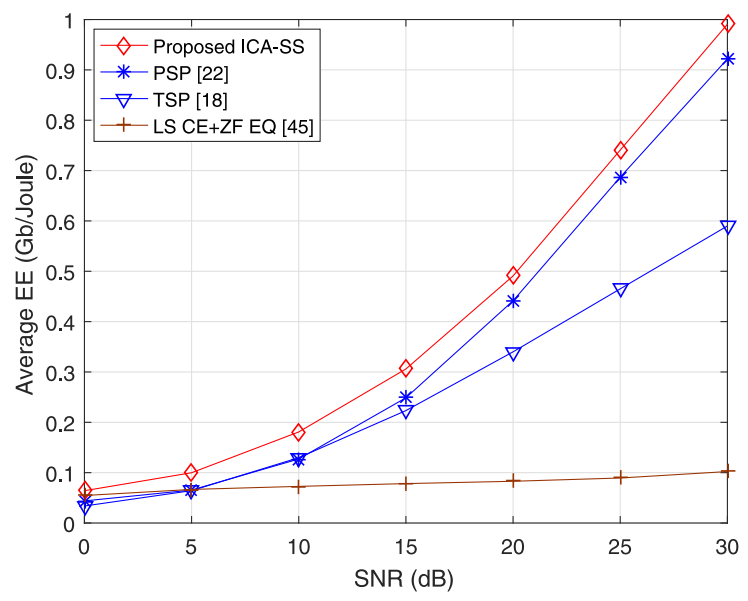

Fig. 9. EE performance of the proposed ICA scheme, in comparison to the existing TSP [18], PSP [22], and LS CE+ZF EQ [45] methods (CE: channel estimation, EQ: equalization).

the time or power is used for wireless power transfer in the TSP [18] and PSP [22]-based self-energy recycling methods.

Fig. 8 shows the throughput of the proposed ICA-SS scheme, compared to TSP [18], PSP [22], and LS CE+ZF EQ [45] schemes. It can be seen that the proposed ICA scheme outperforms the TSP [18] and PSP [22]-based self-energy recycling methods in terms of throughput. It is because those two methods utilize partial resources in either time or power, while the proposed scheme enables full resource utilization for data transmission. Moreover, the proposed ICA-SS scheme exhibits higher throughput than LS CE+ZF EQ [45]. The reason is that the SI is treated as a useful source at destination to enhance the degree of freedom in signal detection or recycle energy in the proposed scheme, while the SI is treated as noise in the LS CE+ZF EQ [45].

Fig. 9 shows the EE of the proposed ICA-SS scheme, compared to TSP [18], PSP [22], and LS CE+ZF EQ [45]. It can be seen that the proposed ICA-SS scheme outperforms LS CE+ZF EQ, TSP, and PSP schemes in terms of EE. The reasons are as follows. 


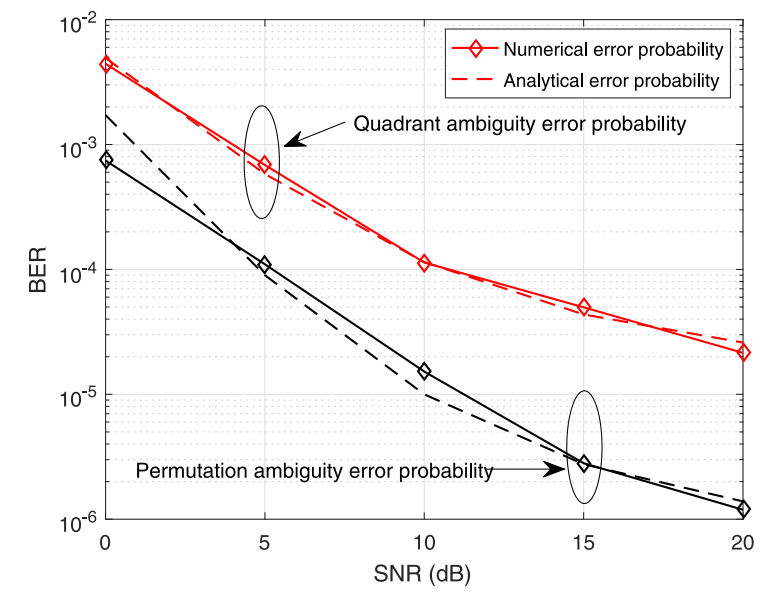

Fig. 10. BER performance due to quadrant and permutation ambiguity with the block length $N_{s}=256$, subcarriers $N=64$.

1) The proposed scheme enables full resource utilization via consecutive data transmission at all time and the same frequency, while the existing methods [18], [22] utilize partial resources in either time or power for data transmission.

2) The proposed scheme utilizes the adaptive SI processing mode selection or recycles harvested energy to enable a much lower power consumption than the existing methods [45] requiring complex SI cancelation procedures.

Fig. 10 shows the BER performance due to quadrant and permutation ambiguity with the block length $N_{s}=256$ and $N=64$ subcarriers. The analytical BER results due to unresolved ambiguity can be approximated by dividing the closedform symbol error probabilities in (48) and (49), which provide a good match with the numerical results. Moreover, as SNR increases, the influence of quadrant and permutation ambiguity on BER performance decreases. At SNR $>10 \mathrm{~dB}$, the BER performance due to quadrant and permutation ambiguity can be lower than $10^{-4}$.

\section{CONCLUSION}

We have proposed an FD AF relay-assisted LLHR IoT system with an adaptive SI processing mode selection strategy at relay and an ICA-SS-based adaptive signal processing scheme at destination. SI was partially canceled at the relay node and the residual SI may be utilized at destination. Therefore, a reduced signal processing cost of SI cancelation and a much higher degree of freedom in signal detection can be achieved. Based on the derived SRSIR thresholds at relay, the proposed strategy of the SI processing mode selection gives aid to the ICA-SS scheme in making an adaptive selection between the ICA (for signal separation and detection) and SS (for signal detection and $\mathrm{EH}$ ) approaches. The simulation results show that, the proposed ICA-SS-based FD $\mathrm{AF}$ relay system achieves higher $\mathrm{EE}$ and throughput than the TSP [18], PSP [22], and LS CE+ZF EQ [45] schemes. It also provides a BER performance that is robust against a wide range of SRSIR, close to the ideal case with perfect CSI and perfect SI cancelation. Furthermore, analytical expressions of the BER of ICA with quadrant ambiguity and permutation ambiguity have been derived, respectively, which match the numerical results very well.

\section{REFERENCES}

[1] H. Duan, Y. Jiang, X. Zhu, Z. Wei, Y. Liu, and L. Gao, "Treating self-interference as source: An ICA assisted full-duplex relay system," in Proc. IEEE Wireless Commun. Netw. Conf. (WCNC), Marrakesh, Morocco, Apr. 2019, pp. 1-6.

[2] N. A. K. Beigi and M. R. Soleymani, "Ultra-reliable energy-efficient cooperative scheme in asynchronous NOMA with correlated sources," IEEE Internet Things J., vol. 6, no. 5, pp. 7849-7863, Oct. 2019.

[3] S. Verma, Y. Kawamoto, Z. M. Fadlullah, H. Nishiyama, and N. Kato, "A survey on network methodologies for real-time analytics of massive IoT data and open research issues," IEEE Commun. Surveys Tuts., vol. 19, no. 3, pp. 1457-1477, 3rd Quart., 2017.

[4] L. Zhao, X. Chi, and Y. Zhu, "Martingales-based energyefficient D-ALOHA algorithms for MTC networks with delay-insensitive/URLLC terminals co-existence," IEEE Internet Things J., vol. 5, no. 2, pp. 1285-1298, Apr. 2018.

[5] H. Guo, J. Liu, J. Zhang, W. Sun, and N. Kato, "Mobile-edge computation offloading for ultradense IoT networks," IEEE Internet Things J., vol. 5, no. 6, pp. 4977-4988, Dec. 2018.

[6] M. Bennis, M. Debbah, and H. V. Poor, "Ultrareliable and low-latency wireless communication: Tail, risk, and scale," Proc. IEEE, vol. 106, no. 10 , pp. 1834-1853, Oct. 2018.

[7] Z. Jiang and S. Mao, "Energy delay tradeoff in multichannel full-duplex wireless LANs," IEEE Internet Things J., vol. 4, no. 3, pp. 658-669, Jun. 2017.

[8] G. Chen, J. P. Coon, A. Mondal, B. Allen, and J. A. Chambers, "Performance analysis for multihop full-duplex IoT networks subject to poisson distributed interferers," IEEE Internet Things J., vol. 6, no. 2, pp. 3467-3479, Apr. 2019.

[9] Z. Wei, X. Zhu, S. Sun, and Y. Huang, "Energy-efficiency-oriented crosslayer resource allocation for multiuser full-duplex decode-and-forward indoor relay systems at $60 \mathrm{GHz}$,' IEEE J. Sel. Areas Commun., vol. 34, no. 12 , pp. 3366-3379, Dec. 2016.

[10] J. Zhou, T.-H. Chuang, T. Dinc, and H. Krishnaswamy, "Integrated wideband self-interference cancellation in the RF domain for FDD and full-duplex wireless," IEEE J. Solid-State Circuits, vol. 50, no. 12, pp. 3015-3031, Dec. 2015.

[11] N. Reiskarimian, M. B. Dastjerdi, J. Zhou, and H. Krishnaswamy, "18.2 highly-linear integrated magnetic-free circulator-receiver for fullduplex wireless," in Proc. IEEE Int. Solid-State Circuits Conf. (ISSCC), San Francisco, CA, USA, Feb. 2017, pp. 316-317.

[12] M. Duarte et al., "Design and characterization of a full-duplex multiantenna system for WiFi networks," IEEE Trans. Veh. Technol., vol. 63, no. 3, pp. 1160-1177, Mar. 2014.

[13] D.-J. van den Broek, E. A. M. Klumperink, and B. Nauta, "An in-band full-duplex radio receiver with a passive vector modulator downmixer for self-interference cancellation,” IEEE J. Solid-State Circuits, vol. 50, no. 12, pp. 3003-3014, Dec. 2015.

[14] Z. Wei, X. Zhu, S. Sun, Y. Huang, L. Dong, and Y. Jiang, "Full-duplex versus half-duplex amplify-and-forward relaying: Which is more energy efficient in 60-GHz dual-hop indoor wireless systems?," IEEE J. Sel. Areas Commun., vol. 33, no. 12, pp. 2936-2947, Dec. 2015.

[15] H. Li et al., "Self-interference cancellation enabling high-throughput short-reach wireless full-duplex communication," IEEE Trans. Wireless Commun., vol. 17, no. 10, pp. 6475-6486, Oct. 2018.

[16] R. Li, Y. Chen, G. Y. Li, and G. Liu, "Full-duplex cellular networks," IEEE Commun. Mag., vol. 55, no. 4, pp. 184-191, Apr. 2017.

[17] S. Huberman and T. Le-Ngoc, "MIMO full-duplex precoding: A joint beamforming and self-interference cancellation structure," IEEE Trans. Wireless Commun., vol. 14, no. 4, pp. 2205-2217, Apr. 2015.

[18] D. Hwang, K. C. Hwang, D. I. Kim, and T.-J. Lee, "Self-energy recycling for RF powered multi-antenna relay channels," IEEE Trans. Wireless Commun., vol. 16, no. 2, pp. 812-824, Feb. 2017.

[19] D. Hwang, S. S. Nam, and J. Yang, "Multi-antenna beamforming techniques in full-duplex and self-energy recycling systems: Opportunities and challenges," IEEE Commun. Mag., vol. 55, no. 10, pp. 160-167, Oct. 2017 . 
[20] V.-D. Nguyen, T. Q. Duong, H. D. Tuan, O.-S. Shin, and H. V. Poor, "Spectral and energy efficiencies in full-duplex wireless information and power transfer," IEEE Trans. Commun., vol. 65, no. 5, pp. 2220-2233, May 2017.

[21] T. Riihonen, L. Zhao, M. Vehkaperä, and X. Wang, "On the feasibility of full-duplex relaying powered by wireless energy transfer," in Proc. IEEE Int. Workshop Signal Process. Adv. Wireless Commun. (SPAWC), Edinburgh, U.K., Jul. 2016, pp. 1-5.

[22] W. Wu, B. Wang, Y. Zeng, H. Zhang, Z. Yang, and Z. Deng, "Robust secure beamforming for wireless powered full-duplex systems with self-energy recycling," IEEE Trans. Veh. Technol., vol. 66, no. 11, pp. 10055-10069, Nov. 2017

[23] C. Zhong, H. A. Suraweera, G. Zheng, I. Krikidis, and Z. Zhang, "Wireless information and power transfer with full duplex relaying," IEEE Trans. Commun., vol. 62, no. 10, pp. 3447-3461, Oct. 2014.

[24] X. Zhou and M. R. McKay, "Secure transmission with artificial noise over fading channels: Achievable rate and optimal power allocation," IEEE Trans. Veh. Technol., vol. 59, no. 8, pp. 3831-3842, Oct. 2010.

[25] J. K. A. Hyvarinen and E. Oja, Independent Component Analysis. New York, NY, USA: Wiley, May 2002.

[26] J. F. Cardoso, "High-order contrasts for independent component analysis," Neural Comput., vol. 11, no. 1, pp. 157-192, Jan. 1999.

[27] Y. Jiang, X. Zhu, E. Lim, Y. Huang, and H. Lin, "Low-complexity semiblind multi-CFO estimation and ICA-based equalization for CoMP OFDM systems," IEEE Trans. Veh. Technol., vol. 63, no. 4 pp. 1928-1934, May 2014.

[28] J. Gao, X. Zhu, and A. K. Nandi, "Non-redundant precoding and PAPR reduction in MIMO OFDM systems with ICA based blind equalization," IEEE Trans. Wireless Commun., vol. 8, no. 6, pp. 3038-3049, Jun. 2009.

[29] J.-H. Lee and O.-S. Shin, "Full-duplex relay based on distributed beamforming in multiuser MIMO systems," IEEE Trans. Veh. Technol., vol. 62, no. 4, pp. 1855-1860, May 2013.

[30] E. Everett, A. Sahai, and A. Sabharwal, "Passive self-interference suppression for full-duplex infrastructure nodes," IEEE Trans. Wireless Commun., vol. 13, no. 2, pp. 680-694, Feb. 2014.

[31] Y. Jin, X.-G. Xia, Y. Chen, and R. Li, "Full-duplex delay diversity relay transmission using bit-interleaved coded OFDM," IEEE Trans. Commun., vol. 65, no. 8, pp. 3250-3258, Aug. 2017.

[32] E. Boshkovska, D. W. K. Ng, N. Zlatanov, and R. Schober, "Practical non-linear energy harvesting model and resource allocation for SWIPT systems," IEEE Commun. Lett., vol. 19, no. 12, pp. 2082-2085, Dec. 2015.

[33] E. Boshkovska, D. W. K. Ng, N. Zlatanov, A. Koelpin, and R. Schober, "Robust resource allocation for MIMO wireless powered communication networks based on a non-linear EH model," IEEE Trans. Commun., vol. 65, no. 5, pp. 1984-1999, May 2017.

[34] A. A. Nasir, X. Zhou, S. Durrani, and R. A. Kennedy, "Relaying protocols for wireless energy harvesting and information processing," IEEE Trans. Wireless Commun., vol. 12, no. 7, pp. 3622-3636, Jul. 2013

[35] R. Tao, A. Salem, and K. A. Hamdi, "Adaptive relaying protocol for wireless power transfer and information processing," IEEE Commun. Lett., vol. 20, no. 10, pp. 2027-2030, Oct. 2016.

[36] Z. Zhou, M. Peng, Z. Zhao, and Y. Li, "Joint power splitting and antenna selection in energy harvesting relay channels," IEEE Signal Process. Lett., vol. 22, no. 7, pp. 823-827, Jul. 2015.

[37] Z. Ding, I. Krikidis, B. Sharif, and H. V. Poor, "Wireless information and power transfer in cooperative networks with spatially random relays," IEEE Trans. Wireless Commun., vol. 13, no. 8, pp. 4440-4453, Aug. 2014.

[38] A. Siligaris et al., "A $60 \mathrm{GHz}$ power amplifier with $14.5 \mathrm{dBm}$ saturation power and 25\% peak PAE in CMOS $65 \mathrm{~nm}$ SOI," IEEE J. Solid-State Circuits, vol. 45, no. 7, pp. 1286-1294, Jul. 2010.

[39] H. Qian, Q. Liu, J. Silva-Martinez, and S. Hoyos, "A 35 dbm output power and $38 \mathrm{~dB}$ linear gain PA with $44.9 \%$ peak PAE at $1.9 \mathrm{GHz}$ in $40 \mathrm{~nm}$ CMOS," IEEE J. Solid-State Circuits, vol. 51, no. 3, pp. 587-597, Mar. 2016

[40] S. Futcha, C. Thibeault, and F. Gagnon, "Predicting CPU requirements with variability equations of ZF-SQRD and rake receiver in a DSP context," in Proc. IEEE Northeast Workshop Circuits Syst. (NEWCAS), Montreal, QC, Canada, Aug. 2007, pp. 1209-1212.

[41] O. Nwamadi, X. Zhu, and A. K. Nandi, "Dynamic physical resource block allocation algorithms for uplink long term evolution," IET Commun., vol. 5, no. 7, pp. 1020-1027, May 2011.

[42] D. Korpi, M. Heino, C. Icheln, K. Haneda, and M. Valkama, "Compact inband full-duplex relays with beyond $100 \mathrm{db}$ self-interference suppression: Enabling techniques and field measurements," IEEE Trans. Antennas Propag., vol. 65, no. 2, pp. 960-965, Feb. 2017.
[43] T. Zhang, C. Su, A. Najafi, and J. C. Rudell, "Wideband dualinjection path self-interference cancellation architecture for full-duplex transceivers," IEEE J. Solid-State Circuits, vol. 53, no. 6, pp. 1563-1576, Jun. 2018.

[44] "TMS320VC33 DSP CPU and instruction set reference guide," Texas Instrum., Houston, TX, USA, Rep. SPRS087E, Jan. 2004. [Online]. Available: http://www.ti.com/lit/ds/symlink/tms320vc33.pdf

[45] D. Darsena, G. Gelli, F. Melito, and F. Verde, "Performance analysis of amplify-and-forward multiple-relay MIMO systems with ZF reception," IEEE Trans. Veh. Technol., vol. 64, no. 7, pp. 3274-3280, Jul. 2015.

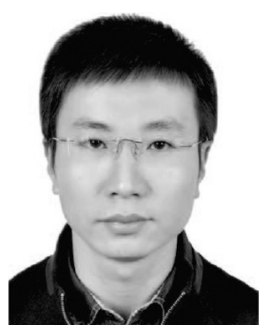

Hanjun Duan (S'19) received the B.S. degree in electronics and information engineering from the College of Applied Science, Jiangxi University of Science and Technology, Ganzhou, China, in 2013, and the M.S. degree in communication and information systems from Yunnan Minzu University, Kunming, China, in 2016. He is currently pursuing the Ph.D. degree with the School of Electrical and Information Engineering, Harbin Institute of Technology, Shenzhen, China.

His research interests include channel estimation and equalization, full-duplex, and blind source separation

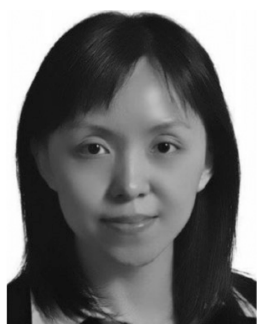

Xu Zhu (S'02-M'03-SM'12) received the B.Eng. degree (Hons.) in electronics and information engineering from the Huazhong University of Science and Technology, Wuhan, China, in 1999, and the Ph.D. degree in electrical and electronic engineering from the Hong Kong University of Science and Technology, Hong Kong, in 2003.

She joined the Department of Electrical Engineering and Electronics, University of Liverpool, Liverpool, U.K., in 2003, as an Academic Member, where she is currently a Reader. She is also with the Harbin Institute of Technology, Shenzhen, China. She has authored or coauthored more than 180 peer-reviewed publications on communications and signal processing. Her research interests include MIMO, channel estimation and equalization, resource allocation, cooperative communications, and green communications.

Dr. Zhu has served as an Editor for the IEEE TRANSACTIONS ON WIRELESS COMMUNICATIONS and a Guest Editor for several international journals such as Electronics. She has acted as a Chair for various international conferences, such as the Vice-Chair of the 2006 and 2008 ICARN International Workshops, the Program Chair of ICSAI 2012, the Symposium Co-Chair of IEEE ICC 2016 and 2019, and the Publicity Chair of IEEE IUCC 2016

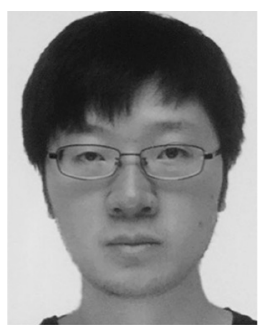

Yufei Jiang (S'12-M'14) received the Ph.D. degree in electrical engineering and electronics from the University of Liverpool, Liverpool, U.K., in 2014.

From 2014 to 2015, he was a Postdoctoral Researcher with the Department of Electrical Engineering and Electronics, University of Liverpool. From 2015 to 2017, he was a Research Associate with the Institute for Digital Communications, University of Edinburgh, Edinburgh, U.K. He is currently an Assistant Professor with the Harbin Institute of Technology, Shenzhen, China. His research interests include $\mathrm{Li}-\mathrm{Fi}$, synchronization, full-duplex, and blind source separation. 


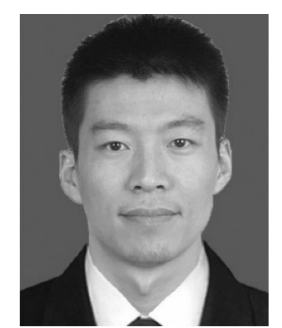

Zhongxiang Wei (S'15-M'17) received the Ph.D. degree in electrical and electronics engineering from the University of Liverpool, Liverpool, U.K., in 2017.

$\mathrm{He}$ is a Research Associate of electrical and electronics engineering with University College London, London, U.K. From March 2016 to March 2017, he was a Research Assistant with the Institution for Infocomm Research, Agency for Science, Technology, and Research (A*STAR), Singapore. From March 2017 to October 2017, he was a visiting student with the Wireless Networks and Communications Group, Harbin Institute of Technology, Shenzhen, China. His research interests include constructive interference design, green communications, full-duplex, millimeterwave communications, and algorithm design.

Dr. Wei was the recipient of the Graduate China National Scholarship Award in 2012 and the A*STAR Research Attachment Programme Studentship in 2016. He has acted as a TPC member or the session chair for various international conferences, such as ICC 2019 and 2020. He was the recipient of Exemplary Reviewer of the IEEE TRANSACTION ON WIRELESS COMMUNICATIONS in 2016.

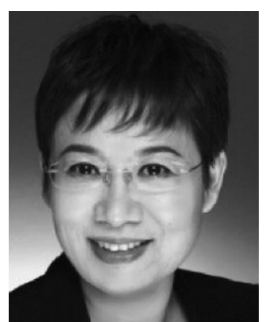

Sumei Sun (F'16) received the B.Sc. degree from Peking University, Beijing, China, the M.Eng. degree from Nanyang Technological University, Singapore, and the Ph.D. degree from the National University of Singapore, Singapore.

She is the Head of the Communications and Networks Department, the Lead Principal Investigator of the Industrial Internet of Things Research Program with the Institute for Infocomm Research, Agency for Science, Technology, and Research, Singapore, and an Adjunct Professor with the National University of Singapore. Her current research interests are in cognitive communications and networks, next-generation wireless communications, and industrial Internet of Things.

Dr. Sun is also serving as a member of the IEEE Communications Society Globecom/ICC Management and Strategy Standing Committee, the Inaugural Editor-in-Chief of the IEEE OPEN JOURNAL OF VEHICUlaR TECHNOLOGY and the IEEE Transactions ON Wireless Communications Steering Committee. She is a Distinguished Speaker of the IEEE Vehicular Technology Society from 2018 to 2021, the Vice Director of the IEEE Communications Society Asia-Pacific Board, and the Chapter Coordinator of the Asia-Pacific Region in the IEEE Vehicular Technologies Society. 